\title{
Rapid clearance of storage-induced micro-erythrocytes alters transfusion recovery
}

Camille Roussel ${ }^{1,3,4,5, *}$, Alexandre Morel ${ }^{1,2,3, *}$, Michael Dussiot ${ }^{1,3, *}$, Mickaël Marin ${ }^{3,4,5}$, Martin Colard $^{1,3}$, Aurélie Fricot-Monsinjon ${ }^{3,4,5}$, Anaïs Martinez ${ }^{1,3}$, Charlotte Chambrion ${ }^{3,4,5}$, Benoît Henry $^{3,4,5}$, Madeleine Casimir ${ }^{1,3}$, Geoffroy Volle ${ }^{3,4,5}$, Mallorie Dépond ${ }^{3,4,5}$, Safi Dokmak ${ }^{6}$, François Paye, Alain Sauvanet ${ }^{6}$, Caroline Le Van Kim ${ }^{3,4,5}$, Yves Colin ${ }^{3,4,5}$, Sonia Georgeault $^{8}$, Philippe Roingeard ${ }^{8,9}$, Steven L. Spitalnik ${ }^{10}$, Papa Alioune Ndour ${ }^{3,4,5}$, Olivier Hermine $^{1,2,3}$, Eldad A. Hod ${ }^{10}$, Pierre A. Buffet ${ }^{3,4,5,11, \xi}$ and Pascal Amireault ${ }^{1,3,4,5, \xi}$

1 Université de Paris, U1163, Laboratory of cellular and molecular mechanisms of hematological disorders and therapeutic implications, INSERM, F-75015 Paris, France

${ }^{2}$ Département d'Hématologie, Assistance Publique - Hôpitaux de Paris, Hôpital Universitaire Necker Enfants Malades, F-75015 Paris, France

${ }^{3}$ Laboratoire d'Excellence GR-Ex, Paris, France

${ }^{4}$ Université de Paris, UMR_S1134, Biologie intégrée du Globule Rouge, INSERM, F-75015 Paris, France

${ }^{5}$ Institut National de la Transfusion Sanguine, F-75015 Paris, France

${ }^{6}$ Department of HBP surgery and Liver Transplantation, Assistance publique - Hôpitaux de Paris, Hôpital Beaujon, F-92110 Clichy, France

${ }^{7}$ Department of digestive surgery, Assistance publique - Hôpitaux de Paris, Hôpital SaintAntoine, F-75012 Paris, France

${ }^{8}$ Plate-Forme IBiSA des Microscopies, PPF ASB, Université de Tours and CHRU de Tours, Tours, France

${ }^{9}$ Inserm U1259 MAVIVH, Université de Tours and CHRU de Tours, Tours, France

${ }^{10}$ Department of Pathology and Cell Biology, Columbia University Irving Medical Center, New York, New York

${ }^{11}$ Assistance Publique - Hôpitaux de Paris, Paris, France

* CR, AMo and MDu share first authorship

$\S$ PAB and PA share last authorship

Running head

SME are cleared from circulation after transfusion

\section{Scientific category}

Transfusion medicine

\section{Corresponding authors:}

Pascal Amireault, Institut National de la Transfusion Sanguine, 6 rue Alexandre Cabanel, F75015 Paris, France, Phone : +33 1444931 37, E-mail: pascal.amireault@inserm.fr and

Pierre A Buffet, Institut National de la Transfusion Sanguine, 6 rue Alexandre Cabanel, F75015 Paris, France, Phone : +33 1444931 13, E-mail: pabuffet@gmail.com

\section{Counts}

Abstract word count: 250

Word count (intro, methods, results, discussion): 4574

Figures: 7

References: 77 


\section{KEY POINTS}

- When storage-induced micro-erythrocytes (SME) are abundant in RBC concentrates, transfusion recovery is diminished.

- SME are rapidly cleared after transfusion, through predominantly spleen- and macrophage-related mechanisms. 


\begin{abstract}
The permanent availability of $\mathrm{RBC}$ for transfusion depends on refrigerated storage, during which morphologically-altered RBC accumulate. Among those, a subpopulation of small RBC, comprising echinocytes III, sphero-echinocytes, and spherocytes, and defined as "storage-induced micro-erythrocytes" (SME) could be rapidly cleared from the circulation after transfusion. We quantified the proportion of SME in RBC concentrates from healthy human volunteers and assessed their correlation with transfusion recovery. We then investigated the fate of SME upon perfusion through human spleens ex vivo. Finally, we explored where and how SME are cleared in a mouse model of blood storage and transfusion. In healthy human volunteers, high proportions of SME in long-stored RBC concentrates correlated with poor transfusion recoveries. When perfused through human spleens, $15 \%$ and $61 \%$ of long-stored RBC and SME were cleared in 70 minutes, respectively. High initial proportions of SME also correlated with high retention of RBC by perfused human spleens. In the mouse model, SME accumulated during storage. Transfusion of long-stored RBC displayed reduced post-transfusion recovery, which was mostly due to clearance of SME. Following transfusions of mice, long-stored RBC accumulated predominantly in the spleen, and were ingested mainly by splenic and hepatic macrophages. In macrophage-depleted mice, splenic accumulation and the clearance of SME were delayed and transfusion recovery was improved. In healthy hosts, SME are cleared predominantly by macrophages in the spleen and liver. When this well-demarcated subpopulation of altered $\mathrm{RBC}$ is abundant in $\mathrm{RBC}$ concentrates, transfusion recovery is diminished. Quantifying SME has the potential to improve blood product quality assessment.
\end{abstract}




\section{INTRODUCTION}

Maintaining the permanent availability of human RBC for transfusion depends on refrigerated storage of RBC concentrates for up to 42 days. The potential impact of storage duration on transfusion safety has been extensively studied. ${ }^{1-5}$ Meta-analyses of prospective randomized controlled trials suggest that there is no advantage in terms of mortality to transfuse shortstored, as compared to standard issue, RBC concentrates. ${ }^{6,7}$ A secondary analysis of the INFORM Trial ${ }^{8}$ concluded that RBC stored for longer than 35 days were not associated with an increased mortality risk; however, no randomized trials have addressed whether the oldest blood is associated with harm. Thus, questions remain regarding the clinical impact of longstored RBC concentrates, in general, and particularly for massive transfusion, with few studies and discordant results ${ }^{9,10}$, or in chronically transfused patients, who are susceptible to transfusion-related iron overload.

The term "storage lesions" refers to the alterations that accumulate during hypothermic storage. ${ }^{11-16}$ The intensity of the storage lesions varies from donor to donor ${ }^{17-20}$ and, despite extensive studies in vitro ${ }^{14,21,22}$, its impact on transfusion outcome remains unclear. The wealth of analyses on RBC transfusion safety sharply contrasts with the small number of studies evaluating the impact of storage duration on transfusion recovery. For example, autologous transfusion of 51-Chromium-labeled RBC in healthy volunteers showed that storage duration is associated with decreased post-transfusion recovery and increased extravascular hemolysis, serum transferrin saturation, and circulating non-transferrin-bound iron. ${ }^{23}$ In addition, in retrospective studies, transfusion-induced hemoglobin increments were significantly decreased when RBC concentrates were stored for longer durations. ${ }^{24-26}$ The 24h-post-transfusion recovery was also significantly decreased when patients with hematological malignancies were transfused with irradiated RBC concentrates stored for more than 2 weeks, as compared to irradiated RBC concentrates stored for less than 2 weeks. ${ }^{17}$ These studies suggest that rapid clearance of long-stored RBC results in reduced transfusion recovery. Early studies showed that some in vitro markers of storage lesions, such as storage hemolysis, correlate poorly with transfusion recovery. ${ }^{27}$ Although other in vitro markers, such as ATP level and the morphology index, showed better correlation with transfusion recovery, the predictive value of these markers is limited. ${ }^{28-35}$ Finally, direct evidence is lacking that RBC with low levels of ATP, or morphologically-altered RBC, are indeed the target for early post-transfusion clearance. 
A well-demarcated subpopulation of morphologically-altered and smaller RBC, comprising echinocytes III, sphero-echinocytes and spherocytes, are easily quantified by imaging flow cytometry in a reproducible, objective and operator-independent manner. ${ }^{36}$ Their decreased surface area, due to progressive membrane loss during storage ${ }^{37-39}$, is expected to lead to their rapid clearance from the recipient's circulation after transfusion. Indeed, the decreased surface area of chemically-treated RBC leads to splenic entrapment. ${ }^{40}$ These small RBC, defined as "storage-induced micro-erythrocytes" (SME) accumulate during storage, reaching a mean proportion of $24 \%$ of the entire RBC population at day 42 of storage. ${ }^{36}$ Rapid clearance of this subpopulation could explain the decreased transfusion recovery observed after transfusion of long-stored RBC concentrates.

Herein, we assessed whether the proportion of SME in stored RBC concentrates correlates with post-transfusion recovery in healthy volunteers. We also investigated the fate of SME during ex vivo perfusion of human spleen, as well as in a specifically developed mouse model. Converging results obtained in human subjects and mice confirmed that SME are rapidly cleared after transfusion, through predominantly spleen- and macrophage-related mechanisms. 


\section{METHODS}

\section{RBC concentrates collection and storage}

Leukoreduced RBC concentrates from healthy donors were stored in saline-adenine-glucosemannitol solution (SAGM) at $2-6^{\circ} \mathrm{C}$ for 42 days and provided by the Etablissement Français du Sang Haut de France-Normandie (French blood banking system). Samples were aseptically collected for each experiment. For human ${ }^{51} \mathrm{Cr}$ RBC post-transfusion recovery studies, leukoreduced RBC concentrates in additive solution-3 (AS-3; $n=30)$ or AS-1 $(n=1)$ were collected from consented healthy volunteers and stored at $2-6^{\circ} \mathrm{C}$. On day $40-42$ of storage, $20-$ $30 \mathrm{ml}$ were aseptically retrieved from the bag to perform ${ }^{51} \mathrm{Cr}$ labeling of $\mathrm{RBC}$ and $24 \mathrm{~h}$-posttransfusion recovery experiments, as described. ${ }^{23,41,42}$ At the same time, a second aliquot of RBC was immediately shipped at $4^{\circ} \mathrm{C}$ from Columbia University to INTS for SME quantification (which occurred 40-48h after the post-transfusion recovery study). Technical details on the RBC concentrates collection are available in supplementary methods.

\section{Scanning electron microscopy on human samples}

Samples were fixed by incubation in $4 \%$ paraformaldehyde, $1 \%$ glutaraldehyde in $0.1 \mathrm{M}$ phosphate buffer ( $\mathrm{pH} 7.3$ ), washed in phosphate-buffer and post-fixed by incubation with $2 \%$ osmium tetroxide for $1 \mathrm{~h}$. Samples were then fully dehydrated in a graded series of ethanol solutions and dried in hexamethyldisilazane. Finally, samples were coated with $4 \mathrm{~nm}$ carbon, using a GATAN PECS 682 apparatus, before observation under a Zeiss Ultra plus FEG-SEM scanning-electron microscope.

\section{Imaging flow cytometry analysis on human samples}

Imaging flow cytometry using ImageStream X Mark II (AMNIS part of EMD Millipore) was performed to determine RBC dimensions and morphology by using brightfield images $(60 \mathrm{X}$ magnification) processed with computer software (IDEAS v6.2, AMNIS) as described. ${ }^{36}$ Technical details are available in supplementary methods.

\section{Human spleen retrieval and ex vivo perfusion}

Spleens were retrieved and processed as described. ${ }^{43}$ All patients underwent distal splenopancreatectomy for pancreatic diseases. Spleens were macroscopically and microscopically normal in all cases. Main splenic artery was cannulated and spleens were 
flushed with cold Krebs-albumin solution for transport to the laboratory. Spleen were coperfused with untreated long-stored RBC (Stained with Celltrace Violet or CellTrace Yellow or $8 \mu \mathrm{g} / \mathrm{ml}$ biotin), rejuvenated long-stored RBC (Stained with carboxyfluorescein succinimidyl ester (CFSE) or Celltrace Far red or $32 \mu \mathrm{g} / \mathrm{ml}$ biotin), and short-stored RBC, at a final hematocrit of $5-30 \%$ (Krebs-albumin solution), over a $70 \mathrm{~min}$ period at $37^{\circ} \mathrm{C}$. Samples were retrieved from the circuit for flow cytometry or imaging flow cytometry analysis. Persistence in circulation was calculated by using the formula: (\% stained RBC in sample/\% stained RBC at T0)*100. Mean retention rate was calculated using formula: 100 - ((Mean \% of stained $\mathrm{RBC}$ in all samples taken between $40-70 \mathrm{~min} / \%$ of stained $\mathrm{RBC}$ at $\mathrm{T} 0$ ) * 100). Rejuvenation was conducted by adding $3 \mathrm{~mL}$ of rejuvenation solution (Zimmer Biomet) to $20 \mathrm{ml}$ of $\mathrm{RBC}$, incubating $1 \mathrm{~h}$ at $37^{\circ} \mathrm{C}$, followed by two washes in saline-adenine-glucosemannitol solution.

\section{Mouse blood banking and transfusion}

Intact, splenectomized, or sham splenectomized C57BL/6 female mice (8-10 weeks) were purchased from Janvier Laboratory. RBC were aseptically collected in CPDA, leukoreduced, and stored at $4^{\circ} \mathrm{C}$ for up to 14 days, as previously reported. ${ }^{44}$ For macrophage depletion, mice were intraperitoneally injected with $1 \mathrm{mg}$ and $0.5 \mathrm{mg}$ of clodronate liposomes (Liposoma), 5 and 2 days before transfusion, respectively. RBC were stained with CFSE $(20 \mu \mathrm{M})$ and washed twice in RPMI. CFSE ${ }^{+}$RBC suspensions $(200 \mu \mathrm{L})$ were transfused through the retroorbital plexus and their proportion after transfusion was measured by flow cytometry. Posttransfusion recovery was calculated using the formula: $\left(\% \mathrm{CFSE}^{+} \mathrm{RBC}\right.$ in sample $/ \% \mathrm{CFSE}^{+}$ $\mathrm{RBC}$ in the $5 \mathrm{~min}$ sample)*100.

\section{Imaging flow cytometry analysis on mouse samples}

Blood bank or transfused mouse samples were diluted (1/100) in DPBS supplemented with glucose $1 \mathrm{~g} / \mathrm{L}$ and BSA $0.01 \%(\mathrm{pH} 7.3)$ and incubated for $50 \mathrm{~min}$ at $37^{\circ} \mathrm{C}$ and analyzed by imaging flow cytometry as for human samples. Transfused RBC were analyzed by gating on $\mathrm{CFSE}^{+}$events. Post-transfusion recovery of the SME subpopulation was calculated using the formula: (post-transfusion recovery) * $\left(\%\right.$ of $\mathrm{CFSE}^{+} \mathrm{RBC}<41 \mu \mathrm{m}^{2}$ in sample/\% of $\mathrm{CFSE}^{+}$ $\mathrm{RBC}<41 \mu \mathrm{m}^{2}$ in the $5 \mathrm{~min}$ sample).

\section{Enrichment in organs}


Spleen and liver were sliced in PBS/2\% FBS/EDTA 2mM while bone marrow cells were obtained by flushing the bones. After a $15 \mathrm{~min}$ incubation at room temperature, supernatants were collected, filtered $(40 \mu \mathrm{m})$ and stained with the DNA stain Draq5. Samples were analyzed by flow cytometry and the enrichment factor was calculated using the following formula: (\% of $\mathrm{CFSE}^{+} \mathrm{RBC}$ within the Draq5 $5^{-}$cells of an organ/\% of $\mathrm{CFSE}^{+} \mathrm{RBC}$ in the blood sample)*100.

\section{In vivo erythrophagocytosis}

Spleen and liver cells were dissociated mechanically using dedicated dissociation kits and gentleMACS dissociator (Miltenyi Biotec). Bone marrow cells were obtained by flushing tibia and femur with $\mathrm{PBS} / 2 \% \mathrm{FBS} / \mathrm{EDTA} 2 \mathrm{mM}$. RBC were removed from the suspension using ACK lysing buffer (Invitrogen). Cells were first incubated with anti-mouse CD16/CD32 clone $2.4 \mathrm{G} 2$ to block IgG receptors (BD). Then, cells were stained at $4^{\circ} \mathrm{C}$ using a panel of antibodies and analyzed by flow cytometry (Supplementary methods).

\section{Statistics}

Calculations for statistical differences between various groups were carried out by ANOVA technique and the Sidak, Tukey, or Kruskall-Wallis posttest to correct for multiple comparisons, as indicated in the figure legends. Spearman test was used to assess correlation between SME and post-transfusion recovery in human volunteers and in ex vivo perfusion of human spleens. Otherwise, a 2-tailed paired Student's t test was used. A $p$-value $<0.05$ was considered statistically significant. Statistical analyses were performed using the Prism software (GraphPad).

\section{Study approval}

The study was conducted according to the Declaration of Helsinki and all research participants provided written, informed consent prior to study participation. The study was approved by the Columbia University Irving Medical Center and Institutional Review Board (IRB \#00001072). Human spleens were retrieved in the context of the "Spleenvivo" project approved by the "Ile-de-France II" IRB on the $4^{\text {th }}$ of September 2017 ( ${ }^{\circ}$ CPP 2015-02-05 SM2 DC). Animal experiments were conducted according to European Directives and were approved by the French Ministry of Education and Research (n¹2071-2017012712012626 v5). 


\section{Data sharing statement}

For original data, please contact pascal.amireault@inserm.fr. 


\section{RESULTS}

\section{Accumulation of morphologically-altered RBC during storage}

At the beginning of storage, scanning electron microscopy images showed that discocytes are abundant and only a few morphologically-altered RBC could be observed (Figure 1A1 and A2). By 41 days of storage, morphologically-altered RBC were abundant and fewer discocytes were observed (Figure 1A3 and A4). RBC shapes, presented according to the Bessis classification $^{45}$, included discocytes, Echinocytes I, II and III, Sphero-Echinocytes and Spherocytes (Figure 1B); the last three categories composing the SME subpopulation. ${ }^{36}$ These qualitative observations confirm the evolution of RBC morphology changes during storage. $^{22,30,46-48}$

\section{Proportions of SME at the end of storage display high inter-donor variability}

We quantified SME weekly throughout storage in $24 \mathrm{RBC}$ concentrates collected from healthy human donors and stored in saline-adenine-glucose-mannitol solution. For all donors, mean SME (range, $\pm \mathrm{SD}$ ) accumulated during storage from $5.2 \%(1.1-9.8, \pm 2.4 \%)$ on Day 3 to $24.6 \%(4.9-68.1, \pm 13.6 \%)$ on Day 42 , with marked inter-donor variability (Figure 2A). We defined low- and high-SME RBC concentrates by cut-off proportions of SME <-1SD and $>+1$ SD at the end of storage, respectively. Low- and high-SME accounted for $12.5 \%$ and $21 \%$ of the RBC concentrates, respectively. These proportions of SME are broadly similar to the proportion of RBC cleared in human post-transfusion recovery studies. ${ }^{20}$

\section{High proportions of SME in long-stored RBC concentrates correlate with poor transfusion recovery in healthy human volunteers}

We correlated pre-transfusion proportions of SME in autologous RBC concentrates (stored in additive solution 3 or additive solution 1 for 42 days) to the $24 \mathrm{~h}$ post-transfusion recovery (using a ${ }^{51}$ Chromium labeling procedure ${ }^{41}$ ) in 31 healthy volunteers. As observed with RBC stored in saline-adenine-glucose-mannitol solution, the projected surface area on normalized frequency plots displayed a bimodal distribution for all donors, enabling quantification of SME in each RBC concentrate (Figure 2B). Mean percentage of SME was $19.9 \%$ and similarly displayed a wide dispersion, with extreme values of $6 \%$ and $52 \%$ (Figure $2 \mathrm{C}$ ). The mean (range, $\pm \mathrm{SD}$ ) $24 \mathrm{~h}$ post-transfusion recovery was $83.5 \%(71.5-95.5, \pm 4.8 \%)$. One of the 31 volunteers had a $24 \mathrm{~h}$ post-transfusion recovery of $71.5 \%$, which was below the FDA 
guideline of $75 \%$. The proportion of pre-transfusion SME negatively correlated with $24 \mathrm{~h}$ post-transfusion recovery $\left(\mathrm{p}=0.02\right.$, spearman $-0.42, \mathrm{r}^{2}=0.24$, Figure $\left.2 \mathrm{C}\right)$. The Mean posttransfusion recovery was significantly decreased $(p=0.03)$ in high-SME RBC concentrates (78\%) when compared to low-SME RBC concentrates (85\%).

\section{Most SME are rapidly cleared upon ex vivo perfusion of human spleens}

We quantified the persistence of long-stored RBC (14 RBC concentrates stored for 35-42 days in saline-adenine-glucose-mannitol solution) mixed with short-stored RBC (stored for 312 days) in the circuit during ex vivo perfusion of human spleens. The morphology of RBC was similar in the perfusion medium and in fresh plasma (Supplementary Figure 1). RBC were, or were not, rejuvenated using a procedure that improves intracellular ATP levels. ${ }^{49}$ Mean proportion of long-stored RBC in the circuit decreased by $14.6 \%(p<0.01)$ in 70 minutes, with marked inter-donor variability (Figure 3A). Rejuvenation restored the RBC projected surface area to a normal gaussian distribution by imaging flow cytometry (similar to that observed at the beginning of storage), decreased the proportion of SME (Figure 3B) and induced an improvement in the circulatory lifespan that reached statistical significance at 1 , $1.5,2,5$ and 40 minutes of perfusion $(p=0.01, p=0.003, p=0.009, p=0.03, p=0.02$, respectively). Proportion of SME was also quantified during ex vivo perfusion. Projected surface area showed a bimodal distribution before perfusion and rapid clearance of SME (Figure 3C). The proportion of SME decreased during perfusion from a mean (range) of $20.0 \%(3.3-36.1 \%)$ initially to $7.8 \%(2.2-16.2 \%)$ at the end $(p=0.02$; Figure $3 \mathrm{D})$. The initial proportion of SME in untreated or rejuvenated RBC concentrates correlated with the mean retention rate in human spleens $(\mathrm{p}=0.03$, Spearman $\mathrm{r}=0.5$; Figure $3 \mathrm{E})$. In contrast, in vitro storage hemolysis, intracellular ATP level, and deformability did not correlate with the retention rate (Supplementary Figure 2).

\section{SME accumulate during storage in a mouse model of RBC storage}

We developed a mouse model of RBC storage and transfusion to assess the fate of SME in vivo and to determine the main mechanisms of RBC clearance. Long-stored $\mathrm{RBC}$ showed a lower $24 \mathrm{~h}$ post-transfusion recovery $(61.9 \pm 2.4 \%)$ as compared to short-stored RBC $(94.7 \pm 0.9 \% ; p<0.001 ;$ Figure $4 A)$.

Morphological alterations of stored mouse RBC were quantified by adapting the imaging flow cytometry approach previously applied to human RBC. Projected surface area showed a 
Gaussian distribution for short-stored and fresh RBC, the latter collected immediately before analysis (Figure 4B). The distribution of long-stored RBC was shifted to the left, indicating that some RBC lost projected surface area (Figure 4C). Mean projected surface area was $47.0 \mu \mathrm{m}^{2}$ and $47.1 \mu \mathrm{m}^{2}$ for fresh and short-stored $\mathrm{RBC}$, respectively; in contrast, it was significantly reduced to $43.0 \mu \mathrm{m}^{2}$ for long-stored $\mathrm{RBC}(\mathrm{p}<0.0001$; Figure 4D). Morphologic analysis of imaging flow cytometry brightfield images categorized long-stored RBC into either altered RBC (echinocytes III, sphero-echinocytes, sphero-stomatocytes, and spherocytes) or normal RBC (echinocytes II, echinocytes I, stomatocytes, and discocytes). As shown in Figure 4E, altered RBC have a mean projected area of $36.5 \mu \mathrm{m}^{2}$ and correspond to $\mathrm{RBC}$ with a lower projected area, whereas $\mathrm{RBC}$ categorized as normal have a profile very similar to that of short-stored RBC. In contrast to stored human RBC, which show a bimodal distribution, the two subpopulations of mouse RBC overlap on the projected area distribution profile in mice. A ROC analysis allowed to determine a gating threshold, set at $41 \mu \mathrm{m}^{2}$, which enables robust identification of altered RBC (Supplementary Figure 3). Using this threshold, the proportion of SME was $13.5 \%$ and $36.1 \%$ in short- and long-stored $\mathrm{RBC}$, respectively $(p<0.001 ;$ Figure 4F).

\section{SME are rapidly cleared from the circulation of transfused mice}

Imaging flow cytometry was used to determine the fate of circulating long-stored RBC posttransfusion. Transfused RBC with reduced surface area were detected in the circulation of recipients $5 \mathrm{~min}$ post-transfusion (Figure $5 \mathrm{~A}$ ). However, at $2 \mathrm{~h}$, an intermediate distribution profile was observed and, at $24 \mathrm{~h}$, the distribution profile of transfused RBC was very similar to that of fresh RBC. SME were progressively cleared from the circulation, declining from $30.5 \%$ at $5 \mathrm{~min}$ post-transfusion, to $20.1 \%(2 \mathrm{~h})$ and $9.3 \%$ at $24 \mathrm{~h}$. When compared to the proportion observed in fresh RBC (10.4\%), the proportion of circulating SME at 5min and $2 \mathrm{~h}$ post-transfusion was significantly increased ( $p<0.0001$ and $p<0.05$, respectively) while it was similar at $24 \mathrm{~h}$ (Figure 5B). The decrease in $24 \mathrm{~h}$ post-transfusion recovery of long-stored RBC was mostly due to the clearance of SME, which had a markedly lower post-transfusion recovery $(16.8 \%)$ than morphologically normal RBC (86.8\%; $<<0.0001$; Figure 5C). Thus, $\sim 75 \%$ of transfused long-stored RBC cleared from the circulation were SME.

Following transfusion, long-stored RBC accumulate predominantly in the spleen and are mainly ingested by macrophages 
The accumulation of transfused RBC was quantified in organs potentially involved in their clearance. Transfused RBC clearance from the circulation was almost linear over a 4-hour period (Figure 5D). Organ accumulation of RBC was measured by dividing the proportion of transfused RBC in organs by the proportion of transfused RBC simultaneously present in the circulation, a ratio expressed as the Enrichment Factor (EF). Long-stored RBC accumulated in the spleen as early as $5 \mathrm{~min}$ after transfusion $(\mathrm{EF}=2.5)$, with a peak at $2 \mathrm{~h}(\mathrm{EF}=3.6)$ followed by a plateau-phase up to $4 \mathrm{~h}(\mathrm{EF}=3.5$; Figure $5 \mathrm{E})$. In the liver, $\mathrm{EF}$ remained low while, in the bone marrow, it reached a maximum after 30min $(\mathrm{EF}=1.4)$. Erythrophagocytic cells in these organs were identified by quantifying their increase in CFSE fluorescence after transfusion. In the spleen, the increase in CFSE fluorescence in red pulp macrophages reached a maximum at 30min (MFI=27092 \pm 5299$)$, then remained strong up to $4 \mathrm{~h}$ post-transfusion $(\mathrm{MFI}=13454 \pm 2295$; Figure 5F1). The maximum increase in CFSE fluorescence was mild in inflammatory monocytes (MFI $=1906 \pm 443$ at $30 \mathrm{~min}$ ), monocytes (MFI=1624 \pm 334 at $30 \mathrm{~min}$ ) and granulocytes $(\mathrm{MFI}=3698 \pm 687$ at $5 \mathrm{~min})$. Liver Kupffer cells also exhibited increased CFSE fluorescence, reaching a maximum at 30 minutes $(\mathrm{MFI}=21023 \pm 6148)$, which declined by $4 \mathrm{~h}$ after transfusion (Figure 5F2). Very mild erythrophagocytosis was detected in the bone marrow (Figure 5F3).

\section{SME clearance is delayed in macrophage-depleted recipients}

In clodronate-treated recipients, the projected surface area of circulating RBC was very similar at $5 \mathrm{~min}$ and $2 \mathrm{~h}$ after transfusion, indicating a delay in clearing $\mathrm{RBC}$ with altered morphology (Figure 6A). At 24h, the distribution profile of RBC transfused into clodronatetreated recipients was very similar to that of fresh RBC. The proportion of SME in circulation was relatively stable over $2 \mathrm{~h}(32.4 \%$ and $28.6 \%$ at $5 \mathrm{~min}$ and $2 \mathrm{~h}$ post-transfusion, respectively), but decreased at 24h (12.4\%; Figure 6B). The proportion of circulating SME at $5 \mathrm{~min}$ and $2 \mathrm{~h}$ was significantly increased $(\mathrm{p}<0.0001$ and $\mathrm{p}<0.001$, respectively) when compared to fresh RBC. $24 \mathrm{~h}$ post-transfusion recovery of long-stored RBC was increased in clodronate-treated recipients $(79.4 \%)$ as compared to controls $(61.9 \%$; $<<0.0001$; Figure $6 \mathrm{C})$. Similarly to control mice (Figure 4C), the SME subpopulation was preferentially cleared (24h post-transfusion recovery $=28.5 \%$ ), whereas the proportion of morphologically normal RBC remained stable $(\mathrm{p}<0.0001$, Figure 6D). PBS liposome-treated recipients demonstrated posttransfusion morphology, SME clearance, and recovery that were similar to what was observed in control recipients (Supplementary Figure 4). 
Organ accumulation and phagocytosis of long-stored RBC and SME in macrophagedepleted or splenectomized mice.

In clodronate-treated recipients, early clearance of long-stored RBC was delayed and significantly reduced at $1 \mathrm{~h}(\mathrm{p}<0.01), 2 \mathrm{~h}$ and $4 \mathrm{~h}$ when compared to controls $(\mathrm{p}<0.0001$; Figure $6 \mathrm{E})$. In the spleen, the EF remained under 1.3 during the first hour post-transfusion and then reached 2.1 at $4 \mathrm{~h}$ (Figure 6F). No enrichment was observed in the liver and bone marrow. In the spleen of these macrophage-depleted recipients, erythrophagocytosis was observed in monocytes and inflammatory monocytes, in particular (Figure 6G1). Similarly, in the liver, strong erythrophagocytosis was observed in monocytes and inflammatory monocytes (Figure 6G2). In the bone marrow, there was only a mild increase in CFSE fluorescence (not shown).

In splenectomized mice, the surface area of transfused RBC returned to normal at the same rate as in controls and sham-splenectomized mice (Figure 7A and Supplementary Figure 4). Accordingly, SME clearance kinetics were similar in splenectomized recipients and controls (Figure 7B). Although SME clearance was not delayed or decreased in splenectomized recipients, the $24 \mathrm{~h}$ post-transfusion recovery of long-stored RBC was increased $(73.7 \%)$ as compared to controls $(61.9 \%, \mathrm{p}<0.001$; Figure $7 \mathrm{C})$. The main determinant of $24 \mathrm{~h}$ posttransfusion recovery was the clearance of SME, which showed a very low post-transfusion recovery (22.5\%) when compared to normal RBC (98.5\%; $<<0.0001$; Figure $7 \mathrm{D})$, indicating that $\sim 96 \%$ of the transfused long-stored RBC cleared from the circulation were SME. Early clearance of long-stored RBC also decreased in splenectomized mice $(p<0.01$, Figure 7E) with low retention observed in the liver and bone marrow (Figure 7F). Early erythrophagocytosis was detected in Kupffer cells and granulocytes (Figure 7G1). Very mild erythrophagocytosis was detected in the bone marrow (Figure 7G2). 


\section{DISCUSSION}

We show that the proportion of small spherocytic RBC that accumulate during storage correlates with post-transfusion RBC recovery in healthy human volunteers (using the "goldstandard" of ${ }^{51}$ Chromium labeling) and with retention rates in human spleens perfused ex vivo. In a mouse RBC storage and transfusion model, we also observe the accumulation of SME and confirm that they are rapidly cleared from the circulation after transfusion. In both humans and mice, the spleen contributes to SME clearance from the circulation. We confirm here that SME progressively accumulate upon storage in commonly-used preservation solutions, and account for $20-25 \%$ of the entire RBC population after 6 weeks of storage. ${ }^{36,50}$ Only a subpopulation of RBC is severely altered during storage, which possibly corresponds to the older RBC component present in the concentrate at the beginning of storage. ${ }^{51,52}$ Based on these observations, the spherocytic shift of stored RBC should be considered as a mechanism of suboptimal transfusion recovery in humans. Thus, quantification of SME becomes a potentially powerful predictor of RBC storage quality and transfusion recovery. Indeed, the proportion of SME at the end of storage displays wide inter-individual variability, which may contribute to the similarly wide variation in post-transfusion recovery observed previously in healthy human volunteers and patients. ${ }^{17,18,20,23}$

A time-dependent decrease in the circulating SME can result from extravascular clearance, intravascular hemolysis, or restoration of morphology (if the SME revert to normal morphology while circulating). In macrophage-depleted mice, SME persisted in the circulation for at least $2 \mathrm{~h}$, suggesting that simple morphological reversibility in the circulation is not a major contributor to the rapid clearance observed in macrophage-intact recipients. We also showed that SME morphology is stable when suspended in fresh plasma.

Post-transfusion recovery results in macrophage-depleted mice suggest that most of the RBC that are cleared from the circulation are SME. The mouse in vivo erythrophagocytosis experiments identify splenic red pulp macrophages and hepatic Kupffer cells as important cells in this post-transfusion phagocytic process. That splenic and hepatic macrophages are the main erythrophagocytic cells in mice agrees with previous reports, ${ }^{53,54}$ along with the increased susceptibility of long-stored RBC to macrophage phagocytosis in vitro. ${ }^{55}$ Little is known about the role of macrophages in clearing transfused RBC in humans, although one study showed increased phagocytosis of long-stored RBC in vitro, ${ }^{56}$ and another suggested that the liver and spleen are the predominant organs involved. ${ }^{57}$ Although it cannot be 
excluded that some long-stored RBC undergo intravascular destruction, converging data from mouse models strongly suggests that, as in humans ${ }^{23}$, the decreased post-transfusion recovery of long-stored RBC depends predominantly on their extravascular hemolysis.

In our murine model, long-stored RBC accumulate in the spleen, likely by mechanical filtration. ${ }^{58}$ The simultaneous accumulation of long-stored RBC in erythrophagocytic organs coupled with their disappearance from circulation suggests that retention of long-stored RBC participates in their elimination. The clearance of morphologically normal RBC, mainly observed in control recipients, could be due to pro-erythrophagocytic conditions induced by the accumulation of transfused RBC in the spleen. This clearance-enhancing mechanism, called the bystander effect, was observed in other situations, such as malaria. ${ }^{59,60} \mathrm{SME}$ clearance is not affected by splenectomy in the mouse, but is delayed by macrophage depletion, indicating that phagocytosis is indispensable; and that the liver, or other organs, like the kidney and lung, that were not investigated here, may compensate for splenic clearance.

Echinocytes III and spheroechinocytes progressively lose membrane content through budding of microvesicles from their spicules. ${ }^{61,62}$ This process also occurs upon exposure of RBC to low $\mathrm{pH}$, low ATP concentrations and high concentrations of calcium. ${ }^{62}$ In the context of pretransfusion refrigerated storage, this shape change is slowed by resuspension of RBC in a large volume of solution. ${ }^{63,64}$ As SME have irreversibly lost more surface than volume upon vesiculation, they no longer display the advantageous surface-to-volume ratio that enables normal discocytes to readily deform and cross the very narrow slits in the spleen. ${ }^{65,66}$ When perfused ex vivo with human RBC, human spleens clear SME from the circuit in two phases. The initial phase occurs within two minutes of perfusion, the second between 10-20min of perfusion. This two-step process suggests the involvement of distinct and successive mechanisms of clearance. RBC adherence to endothelial cells could be involved in the first phase. Previous observations suggested that this type of adherence happens very rapidly ${ }^{67}$, while the timing of the second phase is compatible with a biomechanical retention of SME upstream of narrow splenic slits. Whether the second phase relies predominantly on biomechanical retention or phagocytosis by resident macrophages remains to be determined.

The specific alteration(s) of SME that induce(s) their elimination was not definitely determined. In addition to their pro-adhesive properties (Marin et al., submitted manuscript) and presumed reduced deformability, SME may also over-express pro-phagocytic, "eat me" 
surface markers, such as phosphatidylserine or clustered band 3, or, conversely, under-express "don't eat me" signals, such as CD47. ${ }^{68-70}$ The fact that rejuvenation decreases both the proportion of SME and RBC clearance in the human spleen model also supports the "adherence and biomechanical retention" hypothesis, because rejuvenation reduces adherence of stored RBC to endothelial cells and improves their filterability in a biomimetic splenic filter (Marin et al, submitted manuscript).

The extent of storage lesions varies between human blood donors and mouse strains. ${ }^{18,20,71}$ One possible explanation is that the RBC from good storers may withstand the metabolic stress of storage more robustly than RBC from poor storers. ${ }^{72}$ In addition, storage duration is a very crude and potentially misleading indicator of RBC quality, as "metabolic age" likely differs from "chronological age". In the future, more relevant markers should thus better predict RBC clearance. The $24 \mathrm{~h}$ post-transfusion recovery of ${ }^{51}$ Chromium-labeled $\mathrm{RBC}$ in healthy volunteers is still the gold-standard for FDA approval of any new process for preparing and storing $\mathrm{RBC}$ concentrates ${ }^{20}$, but this method and other analogous methods do not recapitulate all the conditions of a standard allogeneic RBC transfusion. ${ }^{73}$ In addition, post-transfusion recovery studies using ${ }^{51}$ Chromium-labeled RBC are technically challenging, expensive, and only performed at a few centers. Furthermore, the reliability of the method was recently questioned. ${ }^{57}$ Quantification of SME requires access to the Imaging Flow Cytometry technology, but is otherwise a label-free, operator independent, quantitative, reproducible, and reasonably simple method. Data presented here show that $21 \%$ of donors had high SME levels at Day 42, which potentially identifies them as poor storers. Similarly, $12.5 \%$ of donors exhibited low SME levels at Day 42, which may potentially identify them as good storers. This could be relevant for public health because, for example, in France, 3.4\% of RBC concentrates are transfused after Storage Day 35, which represents 78,000 individual transfusions per year. In addition, the proportion of such transfused RBC units may be even higher in the United States, reaching $10-20 \%$ by some estimates. ${ }^{74}$ Moreover, at the median time of RBC concentrates delivery in France (16-20 days of storage), high inter-donor variability is already noticeable.

If a decrease in recovery is not expected to cause serious consequences in the acute transfusion setting, it could have major impact in chronically transfused patients, such as those with sickle cell disease, thalassemia, or low risk myelodysplastic syndrome. For example, transfusion-related iron overload is a major cause of morbidity and mortality in 
these patients, and the need to provide more transfusions because some are of lower quality and recovery could have significant adverse consequences. In the future, quantifying SME could be a surrogate measure for transfusion recovery when assessing promising alternative storage solutions, such as AS- $7^{75}$; new manufacturing processes, such as hypoxic storage ${ }^{76}$; or donor-related factors that contribute to storage quality, such as those identified in REDS-III ${ }^{77}$ and other ${ }^{26}$ studies. These innovations can reduce or abrogate the transfusion of lower quality products, with potential benefit to chronically transfused patients in whom improved transfusion recovery would be expected to decrease the degree of iron overload. 


\section{Acknowledgments}

The authors thank EFS Haut de France-Normandie for providing RBC concentrates. AMo was supported by an Imagine Institute MD-PhD scholarship. CR, MCo, $\mathrm{MCa}$ were supported by a PhD scholarship from the Laboratory of Excellence GR-Ex. CR was supported by a scholarship from the Société française d'hématologie (SFH). This work was supported by State funding from the Agence Nationale de la Recherche under "Investissements d'avenir" program (ANR-10-IAHU-01, ANR-11-LABX-0051 and ANR-18-IDEX-0001), the Fondation Bettencourt Schueller, the National Heart, Lung and Blood Institute grant R01HL133049, HL148151 and by Zimmer Biomet.

\section{Authorship contributions}

CR, AMo, MDu, MC, MM, MCo, AFM, AMa, CC, BH, MCa, GV, MDé, SG, EAH and PA performed experiments. $\mathrm{CR}, \mathrm{AMo}, \mathrm{MDu}, \mathrm{MC}, \mathrm{MM}, \mathrm{MCo}, \mathrm{AFM}, \mathrm{AMa}, \mathrm{CC}, \mathrm{BH}, \mathrm{MCa}, \mathrm{GV}$, MDé, CLVK, YC, SG, PR, SLS, PAN, OH, EAH, PB and PA analyzed the data. SD, FP and AS provided human spleens. CR, AMo, MDu, PAN, SLS, OH, EAH, PB and PA designed the research. CR, AMo, MDu, PB and PA wrote the manuscript. SLS and EAH edited the manuscript. All authors read and approved the manuscript.

\section{Disclosure of Conflict of Interest}

PB and PA are funded in part by Zimmer Biomet. The other authors declare no competing financial interests. 


\section{References}

1. Lacroix J, Hébert PC, Fergusson DA, et al. Age of transfused blood in critically ill adults. N Engl J Med. 2015;372(15):1410-1418.

2. Dhabangi A, Ainomugisha B, Cserti-Gazdewich C, et al. Effect of Transfusion of Red Blood Cells With Longer vs Shorter Storage Duration on Elevated Blood Lactate Levels in Children With Severe Anemia: The TOTAL Randomized Clinical Trial. JAMA. 2015;314(23):2514-2523.

3. Steiner ME, Ness PM, Assmann SF, et al. Effects of red-cell storage duration on patients undergoing cardiac surgery. $N$ Engl J Med. 2015;372(15):1419-1429.

4. Cooper DJ, McQuilten ZK, Nichol A, et al. Age of Red Cells for Transfusion and Outcomes in Critically Ill Adults. N Engl J Med. 2017;377(19):1858-1867.

5. Heddle NM, Cook RJ, Arnold DM, et al. Effect of Short-Term vs. Long-Term Blood Storage on Mortality after Transfusion. N Engl J Med. 2016;375(20):1937-1945.

6. McQuilten ZK, French CJ, Nichol A, Higgins A, Cooper DJ. Effect of age of red cells for transfusion on patient outcomes: a systematic review and meta-analysis. Transfus Med Rev. 2018;32(2):77-88.

7. Chai-Adisaksopha C, Alexander PE, Guyatt G, et al. Mortality outcomes in patients transfused with fresher versus older red blood cells: a meta-analysis. Vox Sang.

2017;112(3):268-278.

8. Cook RJ, Heddle NM, Lee K-A, et al. Red blood cell storage and in-hospital mortality: a secondary analysis of the INFORM randomised controlled trial. Lancet Haematol. 2017;4(11):e544-e552.

9. Cartotto R, Taylor SL, Holmes JH, et al. The Effects of Storage Age of Blood in Massively Transfused Burn Patients: A Secondary Analysis of the Randomized Transfusion Requirement in Burn Care Evaluation Study. Crit Care Med. 2018;46(12):e1097-e1104. 10. Jones AR, Patel RP, Marques MB, et al. Older Blood Is Associated With Increased Mortality and Adverse Events in Massively Transfused Trauma Patients: Secondary Analysis of the PROPPR Trial. Ann Emerg Med. 2019;73(6):650-661.

11. Högman CF, Meryman HT. Storage parameters affecting red blood cell survival and function after transfusion. Transfus Med Rev. 1999;13(4):275-296.

12. Högman CF, Meryman HT. Red blood cells intended for transfusion: quality criteria revisited. Transfusion. 2006;46(1):137-142.

13. Greenwalt TJ. The how and why of exocytic vesicles. Transfusion. 2006;46(1):143152.

14. D'Alessandro A, Kriebardis AG, Rinalducci S, et al. An update on red blood cell storage lesions, as gleaned through biochemistry and omics technologies. Transfusion. 2015;55(1):205-219.

15. Yoshida T, Prudent M, D'alessandro A. Red blood cell storage lesion: causes and potential clinical consequences. Blood Transfus. 2019;17(1):27-52.

16. Hess JR, Greenwalt TG. Storage of red blood cells: new approaches. Transfus Med Rev. 2002;16(4):283-295.

17. Luten M, Roerdinkholder-Stoelwinder B, Schaap NPM, et al. Survival of red blood cells after transfusion: a comparison between red cells concentrates of different storage periods. Transfusion. 2008;48(7):1478-1485.

18. Dern RJ, Gwinn RP, Wiorkowski JJ. Studies on the preservation of human blood. I. Variability in erythrocyte storage characteristics among healthy donors. J Lab Clin Med. 1966;67(6):955-965.

19. Hess JR, Sparrow RL, van der Meer PF, et al. Red blood cell hemolysis during blood bank storage: using national quality management data to answer basic scientific questions. 
Transfusion. 2009;49(12):2599-2603.

20. Dumont LJ, AuBuchon JP. Evaluation of proposed FDA criteria for the evaluation of radiolabeled red cell recovery trials. Transfusion. 2008;48(6):1053-1060.

21. Prudent M, Tissot J-D, Lion N. In vitro assays and clinical trials in red blood cell aging: Lost in translation. Transfus Apher Sci. 2015;52(3):270-276.

22. Bardyn M, Rappaz B, Jaferzadeh K, et al. Red blood cells ageing markers: a multiparametric analysis. Blood Transfus. 2017;15(3):239-248.

23. Rapido F, Brittenham GM, Bandyopadhyay S, et al. Prolonged red cell storage before transfusion increases extravascular hemolysis. J Clin Invest. 2017;127(1):375-382.

24. Rydén J, Clements M, Hellström-Lindberg E, Höglund P, Edgren G. A longer duration of red blood cell storage is associated with a lower hemoglobin increase after blood transfusion: a cohort study. Transfusion. 2019;59(6):1945-1952.

25. Hunsicker O, Hessler K, Krannich A, et al. Duration of storage influences the hemoglobin rising effect of red blood cells in patients undergoing major abdominal surgery. Transfusion. 2018;58(8):1870-1880.

26. Roubinian NH, Plimier C, Woo JP, et al. Effect of donor, component, and recipient characteristics on hemoglobin increments following red blood cell transfusion. Blood.

2019;134(13):1003-1013.

27. Hess JR, Biomedical Excellence for Safer Transfusion (BEST) Collaborative.

Scientific problems in the regulation of red blood cell products. Transfusion.

2012;52(8):1827-1835.

28. Ashby W. THE DETERMINATION OF THE LENGTH OF LIFE OF

TRANSFUSED BLOOD CORPUSCLES IN MAN. $J$ Exp Med. 1919;29(3):267-281.

29. Haradin AR, Weed RI, Reed CF. Changes in physical properties of stored erythrocytes relationship to survival in vivo. Transfusion. 1969;9(5):229-237.

30. Högman CF, de Verdier CH, Ericson A, Hedlund K, Sandhagen B. Studies on the mechanism of human red cell loss of viability during storage at +4 degrees $C$ in vitro. I. Cell shape and total adenylate concentration as determinant factors for posttransfusion survival. Vox Sang. 1985;48(5):257-268.

31. Szymanski IO, Valeri CR, McCallum LE, Emerson CP, Rosenfield RE. Automated differential agglutination technic to measure red cell survival. I. Methodology. Transfusion. 1968;8(2):65-73.

32. Gabrio BW, Donohue DM, Finch CA. Erythrocyte preservation. V. Relationship between chemical changes and viability of stored blood treated with adenosine. $J$ Clin Invest. 1955;34(10):1509-1512.

33. Valeri CR, Pivacek LE, Palter M, et al. A clinical experience with ADSOL preserved erythrocytes. Surg Gynecol Obstet. 1988;166(1):33-46.

34. Heaton WA, Holme S, Smith K, et al. Effects of 3-5 $\log 10$ pre-storage leucocyte depletion on red cell storage and metabolism. Br J Haematol. 1994;87(2):363-368.

35. Reid TJ, Babcock JG, Derse-Anthony CP, et al. The viability of autologous human red cells stored in additive solution 5 and exposed to 25 degrees $\mathrm{C}$ for 24 hours. Transfusion. 1999;39(9):991-997.

36. Roussel C, Dussiot M, Marin M, et al. Spherocytic shift of red blood cells during storage provides a quantitative whole cell-based marker of the storage lesion. Transfusion. 2017;57(4):1007-1018.

37. Lutz HU, Liu SC, Palek J. Release of spectrin-free vesicles from human erythrocytes during ATP depletion. I. Characterization of spectrin-free vesicles. J Cell Biol.

1977;73(3):548-560.

38. Greenwalt TJ, Zehner Sostok C, Dumaswala UJ. Studies in red blood cell preservation. 2. Comparison of vesicle formation, morphology, and membrane lipids during 
storage in AS-1 and CPDA-1. Vox Sang. 1990;58(2):90-93.

39. Kriebardis AG, Antonelou MH, Stamoulis KE, et al. RBC-derived vesicles during storage: ultrastructure, protein composition, oxidation, and signaling components.

Transfusion. 2008;48(9):1943-1953.

40. Safeukui I, Buffet PA, Deplaine G, et al. Quantitative assessment of sensing and sequestration of spherocytic erythrocytes by the human spleen. Blood. 2012;120(2):424-430.

41. Moroff G, Sohmer PR, Button LN. Proposed standardization of methods for determining the 24-hour survival of stored red cells. Transfusion. 1984;24(2):109-114.

42. Bitan ZC, Zhou A, McMahon DJ, et al. Donor Iron Deficiency Study (DIDS): protocol of a study to test whether iron deficiency in blood donors affects red blood cell recovery after transfusion. Blood Transfus. 2019;17(4):274-280.

43. Buffet PA, Milon G, Brousse V, et al. Ex vivo perfusion of human spleens maintains clearing and processing functions. Blood. 2006;107(9):3745-3752.

44. Fischer D, Büssow J, Meybohm P, Zacharowski K, Jennewein C. Novel method to leukoreduce murine blood for transfusion: how to reduce animal usage. Transfusion. 2016;56(1):146-152.

45. Bessis M. Red cell shapes. An illustrated classification and its rationale. Nouv Rev Fr Hematol. 1972;12(6):721-745.

46. Berezina TL, Zaets SB, Morgan C, et al. Influence of storage on red blood cell rheological properties. J Surg Res. 2002;102(1):6-12.

47. D'Alessandro A, D'Amici GM, Vaglio S, Zolla L. Time-course investigation of SAGM-stored leukocyte-filtered red bood cell concentrates: from metabolism to proteomics. Haematologica. 2012;97(1):107-115.

48. Blasi B, D'Alessandro A, Ramundo N, Zolla L. Red blood cell storage and cell morphology. Transfus Med. 2012;22(2):90-96.

49. D’Alessandro A, Gray AD, Szczepiorkowski ZM, et al. Red blood cell metabolic responses to refrigerated storage, rejuvenation, and frozen storage. Transfusion.

2017;57(4):1019-1030.

50. Roussel C, Monnier S, Dussiot M, et al. Fluorescence Exclusion: A Simple Method to Assess Projected Surface, Volume and Morphology of Red Blood Cells Stored in Blood Bank. Front Med (Lausanne). 2018;5:164.

51. Tuo W-W, Wang D, Liang W-J, Huang Y-X. How cell number and cellular properties of blood-banked red blood cells of different cell ages decline during storage. PLoS One. 2014;9(8):e105692.

52. Mykhailova O, Olafson C, Turner TR, D'Alessandro A, Acker JP. Donor-dependent aging of young and old red blood cell subpopulations: Metabolic and functional heterogeneity. Transfusion. 2020;60(11):2633-2646.

53. Hod EA, Zhang N, Sokol SA, et al. Transfusion of red blood cells after prolonged storage produces harmful effects that are mediated by iron and inflammation. Blood. 2010;115(21):4284-4292.

54. Hudson KE, de Wolski K, Kapp LM, et al. Antibodies to Senescent Antigen and C3 Are Not Required for Normal Red Blood Cell Lifespan in a Murine Model. Front Immunol. 2017;8:1425.

55. Wojczyk BS, Kim N, Bandyopadhyay S, et al. Macrophages clear refrigerator storagedamaged red blood cells and subsequently secrete cytokines in vivo, but not in vitro, in a murine model. Transfusion. 2014;54(12):3186-3197.

56. Veale MF, Healey G, Sparrow RL. Longer storage of red blood cells is associated with increased in vitro erythrophagocytosis. Vox Sang. 2014;106(3):219-226.

57. Francis RO, Mahajan S, Rapido F, et al. Reexamination of the chromium-51-labeled posttransfusion red blood cell recovery method. Transfusion. 2019;59(7):2264-2275. 
58. MacDonald IC, Schmidt EE, Groom AC. The high splenic hematocrit: a rheological consequence of red cell flow through the reticular meshwork. Microvasc Res. 1991;42(1):60 76.

59. Douglas NM, Anstey NM, Buffet PA, et al. The anaemia of Plasmodium vivax malaria. Malar J. 2012;11:135.

60. White NJ. Anaemia and malaria. Malar J. 2018;17(1):371.

61. Laczkó J, Szabolcs M, Jóna I. Vesicle release from erythrocytes during storage and failure of rejuvenation to restore cell morphology. Haematologia (Budap). 1985;18(4):233248.

62. Hess JR. Red cell changes during storage. Transfus Apher Sci. 2010;43(1):51-59.

63. Meryman HT, Hornblower M, Syring R, Mesbah-Karimi N. Extending the storage of red cells at 4 degrees C. Transfus Sci. 1994;15(2):105-115.

64. Meryman HT. Quarantine of red blood cells by long-term storage in the liquid phase. Transfus Clin Biol. 1994;1(3):188-191.

65. Perrotta S, Gallagher PG, Mohandas N. Hereditary spherocytosis. Lancet. 2008;372(9647):1411-1426.

66. Waugh RE, Sarelius IH. Effects of lost surface area on red blood cells and red blood cell survival in mice. Am. J. Physiol. 1996;271(6 Pt 1):C1847-1852.

67. Safeukui I, Correas J-M, Brousse V, et al. Retention of Plasmodium falciparum ringinfected erythrocytes in the slow, open microcirculation of the human spleen. Blood. 2008;112(6):2520-2528.

68. Verhoeven AJ, Hilarius PM, Dekkers DWC, Lagerberg JWM, de Korte D. Prolonged storage of red blood cells affects aminophospholipid translocase activity. Vox Sang. 2006;91(3):244-251.

69. Azouzi S, Romana M, Arashiki N, et al. Band 3 phosphorylation induces irreversible alterations of stored red blood cells. Am. J. Hematol. 2018;93(5):E110-E112.

70. Anniss AM, Sparrow RL. Expression of CD47 (integrin-associated protein) decreases on red blood cells during storage. Transfus. Apher. Sci. 2002;27(3):233-238.

71. Zimring JC, Smith N, Stowell SR, et al. Strain-specific red blood cell storage, metabolism, and eicosanoid generation in a mouse model. Transfusion. 2014;54(1):137-148. 72. D'Alessandro A, Zimring JC, Busch M. Chronological storage age and metabolic age of stored red blood cells: are they the same? Transfusion. 2019;59(5):1620-1623.

73. Roussel C, Buffet PA, Amireault P. Measuring Post-transfusion Recovery and Survival of Red Blood Cells: Strengths and Weaknesses of Chromium-51 Labeling and Alternative Methods. Front Med (Lausanne). 2018;5:130.

74. Glynn SA, Klein HG, Ness PM. The red blood cell storage lesion: the end of the beginning. Transfusion. 2016;56(6):1462-1468.

75. Cancelas JA, Dumont LJ, Maes LA, et al. Additive solution-7 reduces the red blood cell cold storage lesion. Transfusion. 2015;55(3):491-498.

76. Dumont LJ, Yoshida T, AuBuchon JP. Anaerobic storage of red blood cells in a novel additive solution improves in vivo recovery. Transfusion. 2009;49(3):458-464.

77. Lanteri MC, Kanias T, Keating S, et al. Intradonor reproducibility and changes in hemolytic variables during red blood cell storage: results of recall phase of the REDS-III RBC-Omics study. Transfusion. 2019;59(1):79-88. 


\section{FIGURE LEGENDS}

Figure 1: Accumulation of morphologically-altered RBC during storage. (A) A shortstored RBC sample (day 12 of storage) containing a majority of Discocytes, Echinocytes I or II ; in (A1) panoramic view (2000x) and (A2) detail of the same sample (5000x) and a longstored RBC sample (day 41 of storage) containing abundant Echinocytes III, Spheroechinocytes and Spherocytes in (A3) panoramic view (2000x) and (A4) detail of the same sample (5000x). (B) Representative scanning electron images of RBC shapes observed during storage in Saline-Adenine-Glucose-Mannitol solution (SAGM): Discocytes (D), Echinocytes I, II and III (EI, EII, EIII), Sphero-Echinocytes (SE) and Spherocytes (S), numerically zoomed regions from 2000x acquisitions. The red square highlights morphologically-altered $\mathrm{RBC}$ defined as storage-induced micro-erythrocytes (SME). Scale bars $=2 \mu \mathrm{M}$

Figure 2: The proportion of storage-induced micro-erythrocytes (SME) at the end of storage correlates with $24 \mathrm{~h}$ post-transfusion recovery in healthy human volunteers. (A) Quantification of SME upon storage of RBC concentrates (RCC) in saline-adenine-glucosemannitol solution $(n=24)$ between day 3 and day 42 (mean value in solid black line). Low (blue lines) and high proportion of SME (red lines) were defined by a proportion of SME <1SD (11\%) and $>+1 \mathrm{SD}(38 \%)$ at the end of storage, respectively. (B) Representative normalized frequency plot for a RBC concentrate at the end of storage in additive solution-3 (AS-3), showing a well-demarcated subpopulation of SME. The subpopulation of SME contains spherocytes, spheroechinocytes, and echinocytes III (insert i), whereas normal-sized RBC (insert ii) contain discocytes, echinocytes I, and echinocytes II. (C) Correlation between the $24 \mathrm{~h}$ post-transfusion recovery and the proportions of SME quantified by imaging flow cytometry at the end of storage $\left(n=31, p=0.02\right.$, spearman $\left.-0.42, r^{2}=0.24\right)$.

Figure 3: SME are rapidly cleared when perfused through human spleens ex vivo. (A) Kinetics (mean +/- SEM) of the normalized concentration in the perfusate of human spleens ex vivo $(\mathrm{n}=7)$ of $14 \mathrm{RBC}$ concentrates stored for 35-42 days and rejuvenated (RW, dashed line) or not (NT, full line). (B) Representative normalized frequency plot of the projected surface area of RBC stored for 42 days before (solid gray histogram) and after (dashed line) rejuvenation. The dashed vertical line defines the gating cutoff for SME. (C) Representative frequency plot of projected surface area of stored RBC (37 days) before (dark grey histogram), and at different time points after, perfusion through a human spleen ex vivo (0 $\min =$ solid light grey line, $2 \mathrm{~min}=$ solid black line, $20 \mathrm{~min}=$ dotted black line, and $40 \mathrm{~min}=$ dashed black line). (D) Proportion of SME at the beginning (T0) and mean proportion of SME at all observations between 40-70 min (T40-70 min) of perfusion through human spleens ex vivo $(\mathrm{n}=6$; red dashed line represents the mean). (E) Correlation between mean retention rate in human spleens perfused ex vivo and the proportion of SME in the RBC concentrate before transfusion $\left(\mathrm{n}=28, \mathrm{p}=0.03\right.$, spearman $\left.0.5, \mathrm{r}^{2}=0.2\right)$. In A, a Sidak's multiple comparisons test was used to compare, at each time point, the persistence in circulation of rejuvenated vs non treated $\mathrm{RBC}\left(* * \mathrm{p}<0.01,{ }^{*} \mathrm{p}<0.05\right)$. In $\mathrm{D}$, a paired student $\mathrm{t}$ test was used.

Figure 4: Identification and quantification of a subpopulation of SME in a mouse model. (A) Post-transfusion recovery of long-stored RBC ( 14 days of storage, in grey, $n=7$ ) is decreased at $2 \mathrm{~h}$ and $24 \mathrm{~h}$ after transfusion to control recipients, as compared to short-stored RBC ( 1 day of storage, in red, $n=7$ ). (B) Normalized frequency plots of projected surface area for fresh (black line, $n=11$ ) and short-stored RBC (red line, $n=9$ ) show similar patterns. (C) Long-stored $\mathrm{RBC}$ (grey line, $\mathrm{n}=9$ ) have a reduced projected surface area when compared to 
short-stored RBC (red line). (D) Quantification of projected surface area of front views of focused $\mathrm{RBC}$, obtained by imaging flow cytometry, shows a significant decrease in longstored RBC. (E) Representative analysis of brightfield images of long-stored RBC show that morphologically-altered RBC (dotted black line) have a reduced projected surface area when compared to RBC with normal morphology (black line). Morphologically-altered RBC were defined as echinocytes III, spheroechinocytes, spherostomatocytes, and spherocytes, whereas normal RBC comprise discocytes, echinocytes I, and echinocytes II. (F) SME in short-stored and long-stored RBC accumulate during storage. Data are presented as mean \pm SEM. In A, a Sidak's multiple comparisons test was used to compare, at each time point, the recovery of short-stored $v s$ long-stored RBC. In $\mathrm{D}, * * * * \mathrm{p}<0.0001, \mathrm{~ns}=$ not significant, by Tukey's multiple comparison. In $\mathrm{F}, * * * \mathrm{p}<0.001$ by student $\mathrm{t}$ test.

Figure 5: In the mouse model, SME are rapidly cleared, accumulate in the spleen, and are processed predominantly by macrophages. (A) Representative normalized frequency plot of projected surface area for long-stored mouse RBC, as observed at $5 \mathrm{~min}$ (green line), $2 \mathrm{~h}$ (yellow line), and 24h (red line) after transfusion to a syngeneic C57Bl/6 mouse. Control fresh RBC from a non-transfused mouse (blue) are shown as a reference. The dashed white vertical line defines the gating of SME. (B) Declining proportion of SME in the circulation following transfusion ( $\mathrm{n}=10$ mice/group). (C) Variable persistence in the circulation of longstored RBC (grey line), that contain the 2 complementary subpopulations of SME (black dashed line) and morphologically normal RBC (black full line), computed by combining the flow cytometry and Imagestream data (10 mice/group). (D) Proportion of long-stored RBC that were cleared 5-240 minutes post-transfusion ( $\mathrm{n}=8$ mice/time point). (E) Enrichment factor 5-240 minutes post-transfusion (ratio of transfused $\mathrm{CFSE}^{+} \mathrm{RBC}$ in a sliced organ/ $\mathrm{CFSE}^{+} \mathrm{RBC}$ in venous blood, $\mathrm{n}=4$ mice/time point), in the spleen (red line), liver (black line), and bone marrow (blue line). (F1-3) Post-transfusion erythrophagocytosis of RBC in the spleen (F1), liver (F2), and bone marrow (F3), estimated by the increase in CFSE median fluorescence intensity (MFI) of macrophages (red lines), monocytes (blue lines), inflammatory monocytes (purple lines), and granulocytes (black lines), when compared to control non-transfused mice ( $\mathrm{n}=3$ mice/time point). Data are presented as mean \pm SEM. In $\mathrm{B}$, $* * * * \mathrm{p}<0.0001, * \mathrm{p}<0.05$, ns $=$ not significant, when compared to the "Fresh $\mathrm{RBC}$ " condition by a Kruskall-Wallis test. In C, a Sidak's multiple comparisons test was used to compare, at each time point, the recovery of the SME subpopulation $v s$ normal subpopulation.

Figure 6: Clearance kinetics of SME in clodronate-treated mice. (A) Representative normalized frequency plots of projected surface area for long-stored mouse RBC, as observed $5 \mathrm{~min}$ (green line), $2 \mathrm{~h}$ (yellow line), and $24 \mathrm{~h}$ (red line) after transfusion of a clodronate-treated mouse. Control fresh RBC from a non-transfused mouse (blue) are shown as a reference. The dashed white vertical line defines gating of SME. (B) Delayed clearance of SME in the circulation following transfusion in clodronate-treated mice ( $\mathrm{n}=10$ in each group). (C) Posttransfusion recovery of long-stored $\mathrm{RBC}$ is increased at $2 \mathrm{~h}$ and $24 \mathrm{~h}$ after transfusion to clodronate-treated recipients (dashed line, $n=10$ ), as compared to controls (full line, $n=10$ ). (D) Variable persistence in circulation of long-stored RBC (grey line), that contain the two complementary subpopulations of SME (black dashed line) and morphologically normal RBC (black full line), computed by combining the flow cytometry and Imagestream data following transfusion in clodronate-treated mice ( $\mathrm{n}=10$ in each group). (E) Decreased proportion of long-stored RBC that were cleared 60-240 minutes post-transfusion in clodronate-treated recipients (dashed line, $\mathrm{n}=6$ mice/time point) when compared to controls (full line, $\mathrm{n}=8$ mice/time point). (F) Enrichment factor (ratio of transfused $\mathrm{CFSE}^{+} \mathrm{RBC}$ in a sliced organ/CFSE ${ }^{+} \mathrm{RBC}$ in venous blood) 5-240 minutes post-transfusion, in the spleen (red line), 
liver (black line), and bone marrow (blue line) following transfusion in clodronate-treated mice ( $\mathrm{n}=3$ mice/time point). Post-transfusion erythrophagocytosis of $\mathrm{RBC}$ in the spleen (G1) and liver (G2), estimated by the increase in median fluorescence intensity (MFI) of CFSE in macrophages (red lines), monocytes (blue lines), inflammatory monocytes (purple lines) and granulocytes (black lines) in clodronate-treated recipients when compared to control nontransfused mice ( $\mathrm{n}=3$ mice/time point). Data are presented as mean $\pm \mathrm{SEM}$. In $\mathrm{B},{ }^{* * *} \mathrm{p}<0.001$, $* * * * \mathrm{p}<0.0001$, ns $=$ not significant, when compared to the "Fresh RBC" condition by a Kruskall-Wallis test. In C (and E), a Sidak's multiple comparisons test was used to compare, at each time point, the recovery (clearance) in clodronate-treated $v s$ control recipients $\left({ }^{* *} \mathrm{p}<0.01\right)$. In $\mathrm{D}$, a Sidak's multiple comparisons test was used to compare, at each time point, the recovery of the SME subpopulation vs normal subpopulation.

Figure 7: Clearance kinetics of SME in splenectomized mice. (A) Typical normalized frequency plots of projected surface area for long-stored mouse RBC, as observed $5 \mathrm{~min}$ (green line), $2 \mathrm{~h}$ (yellow line), and $24 \mathrm{~h}$ (red line) after transfusion to a splenectomized mouse. Control fresh RBC from a non-transfused mouse (blue) are shown as a reference. The dashed white vertical line defines gating of SME. (B) Delayed clearance of SME in the circulation following transfusion in splenectomized mice $(\mathrm{n}=10$ in each group). (C) Post-transfusion recovery of long-stored $\mathrm{RBC}$ is increased at $24 \mathrm{~h}$ after transfusion to splenectomized recipients (dashed line, $\mathrm{n}=10$ ), as compared to controls (full line, $\mathrm{n}=10$ ). (D) Variable persistence in circulation of long-stored RBC (grey line), that contain the two complementary subpopulations of SME (black dashed line) and morphologically normal RBC (black full line), computed by combining the flow cytometry and Imagestream data following transfusion in splenectomized mice ( $\mathrm{n}=10$ in each group). (E) Decreased proportion of long-stored $\mathrm{RBC}$ that were cleared at 240 minutes post-transfusion in splenectomized recipients (dashed line, $\mathrm{n}=6$ mice/time point) when compared to controls (full line, $\mathrm{n}=8$ mice/time point). ( $\mathrm{F}$ ) Enrichment factor (ratio of transfused $\mathrm{CFSE}^{+} \mathrm{RBC}$ in a sliced organ/CFSE ${ }^{+} \mathrm{RBC}$ in venous blood) 5-240 minutes post-transfusion, in the liver (black line) and bone marrow (blue line) following transfusion in splenectomized mice $(\mathrm{n}=3$ mice/time point). Post-transfusion erythrophagocytosis of RBC in the liver (G1) and bone marrow (G2), estimated by the increase in MFI of CFSE in splenectomized recipients $(n=3$ mice/time point). Data are presented as mean \pm SEM. In $\mathrm{B} * * * * \mathrm{p}<0.0001,{ }^{*} \mathrm{p}<0.05$, ns $=$ not significant, when compared to the "Fresh RBC" condition by a Kruskall-Wallis test. In C (and E), a Sidak's multiple comparisons test was used to compare, at each time point, the recovery (clearance) in splenectomized $v s$ control recipients $(* * * p<0.001)$. In D, a Sidak's multiple comparisons test was used to compare, at each time point, the recovery of the SME subpopulation $v s$ normal subpopulation. 
A

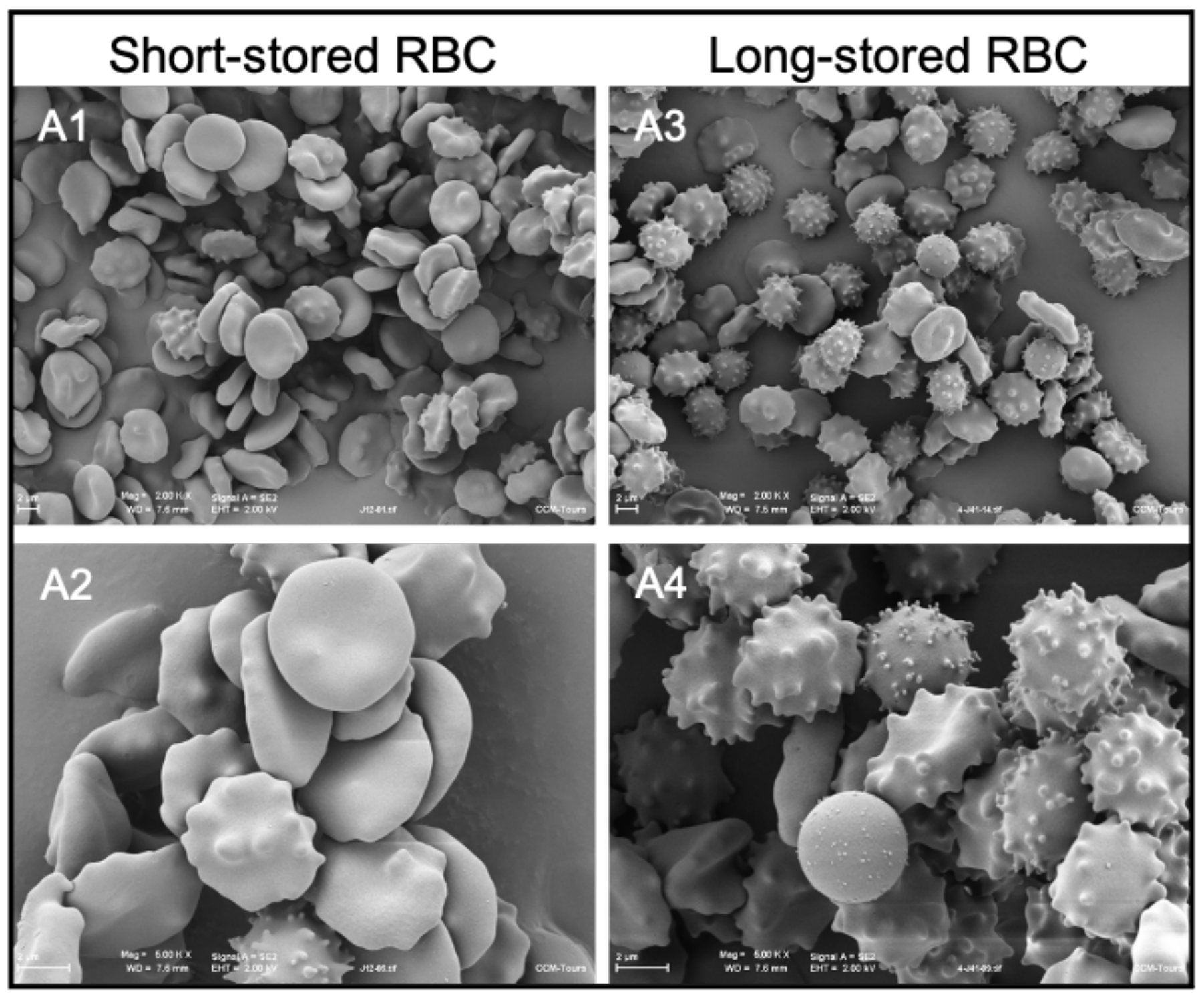

B
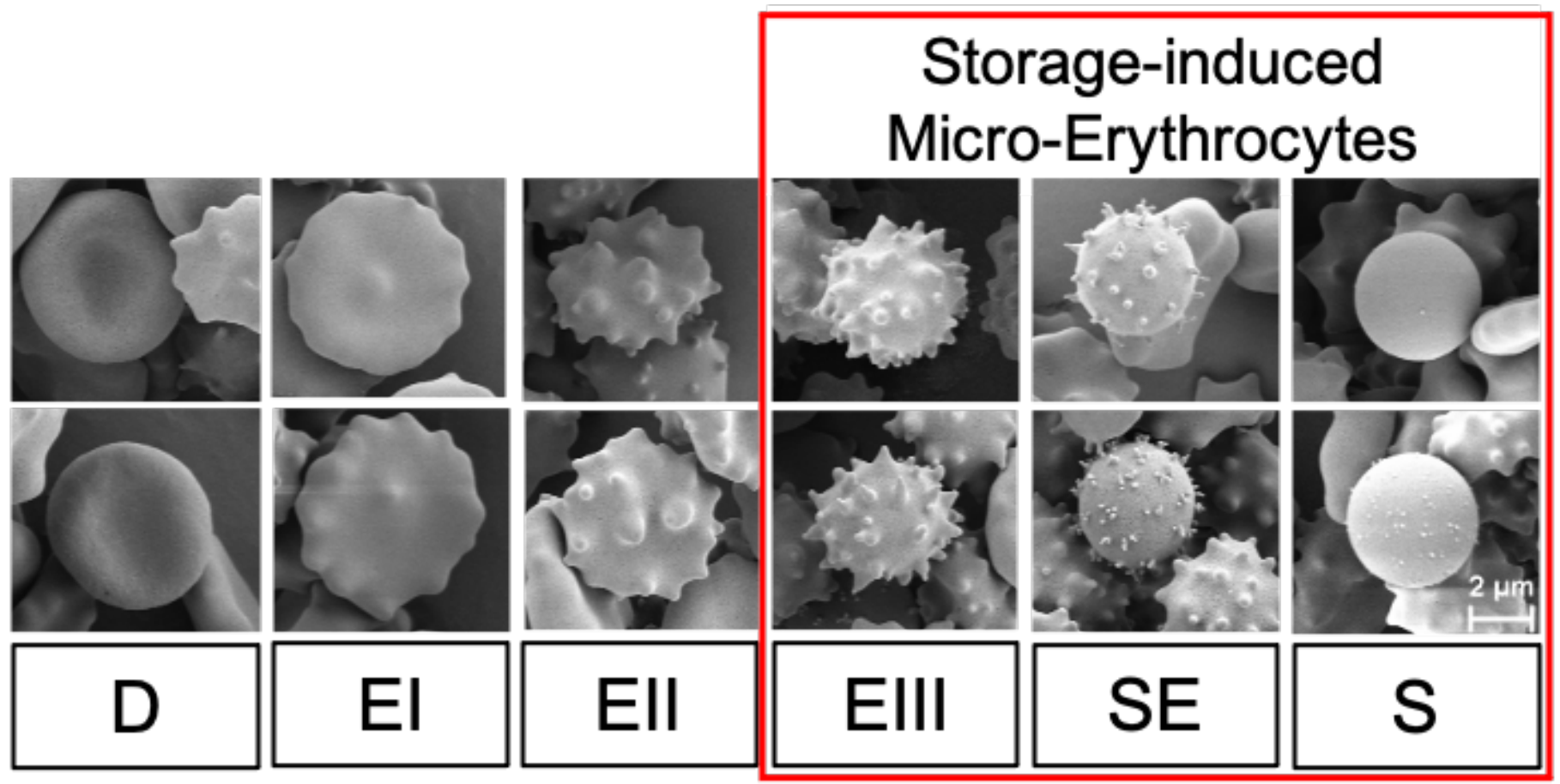

Figure 1 
A

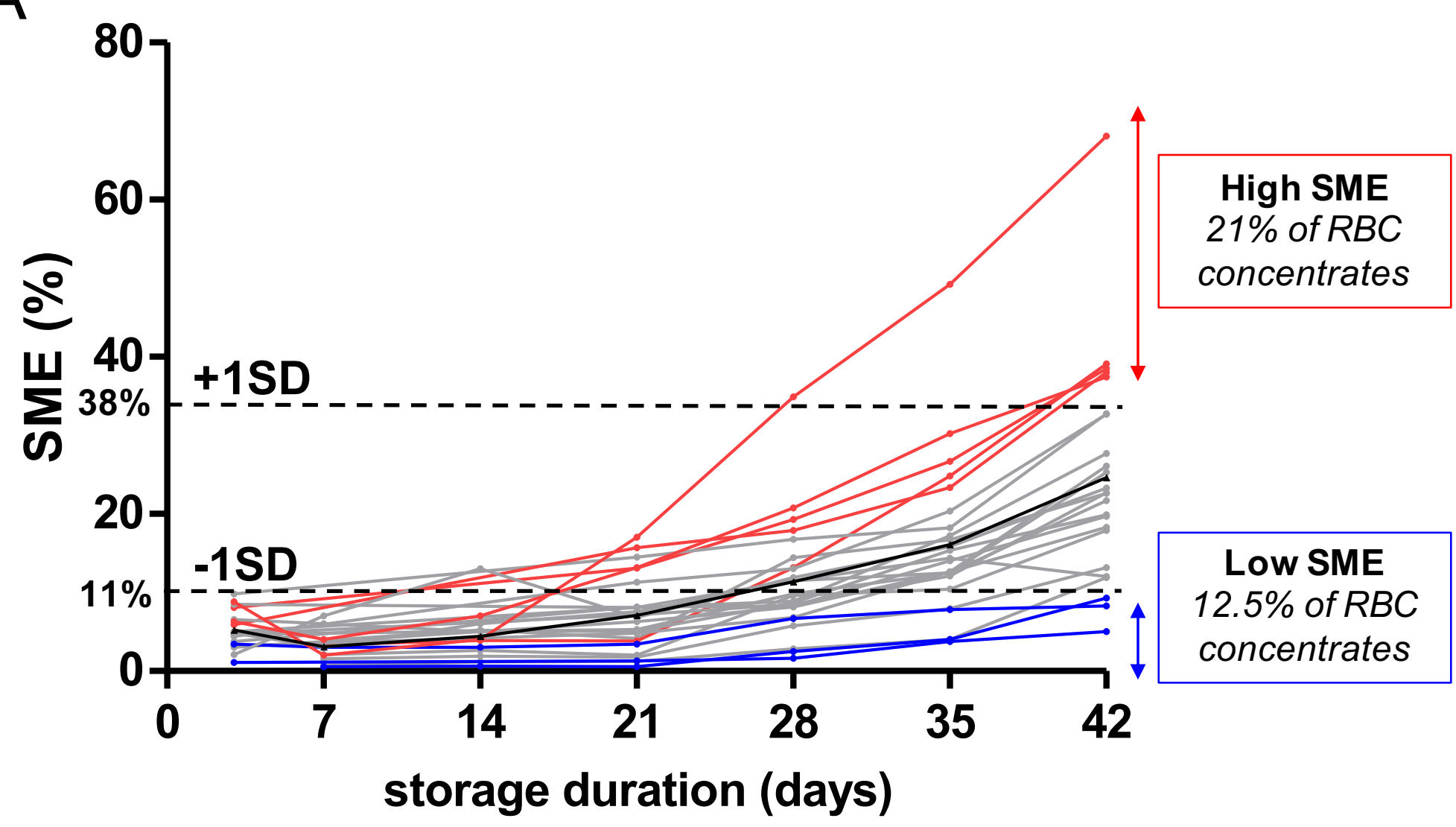

B
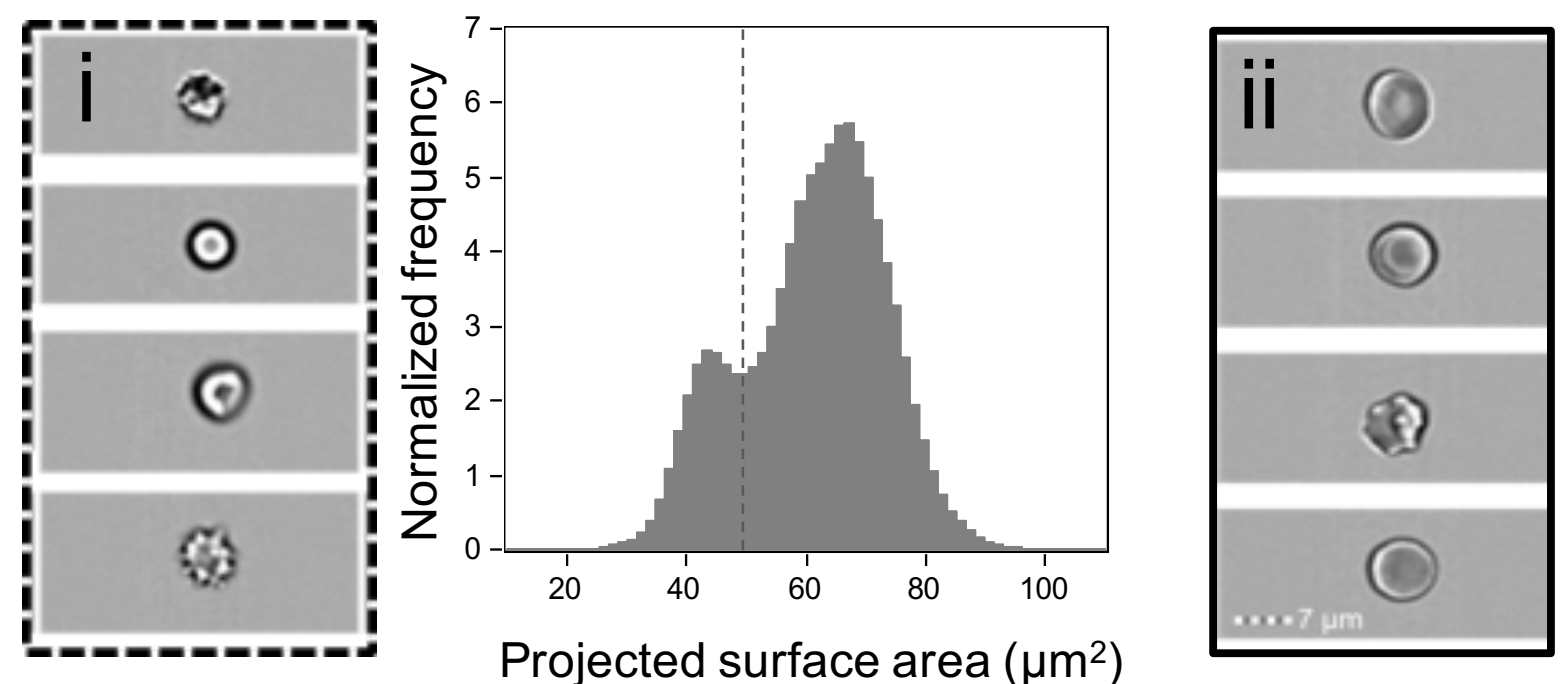

C

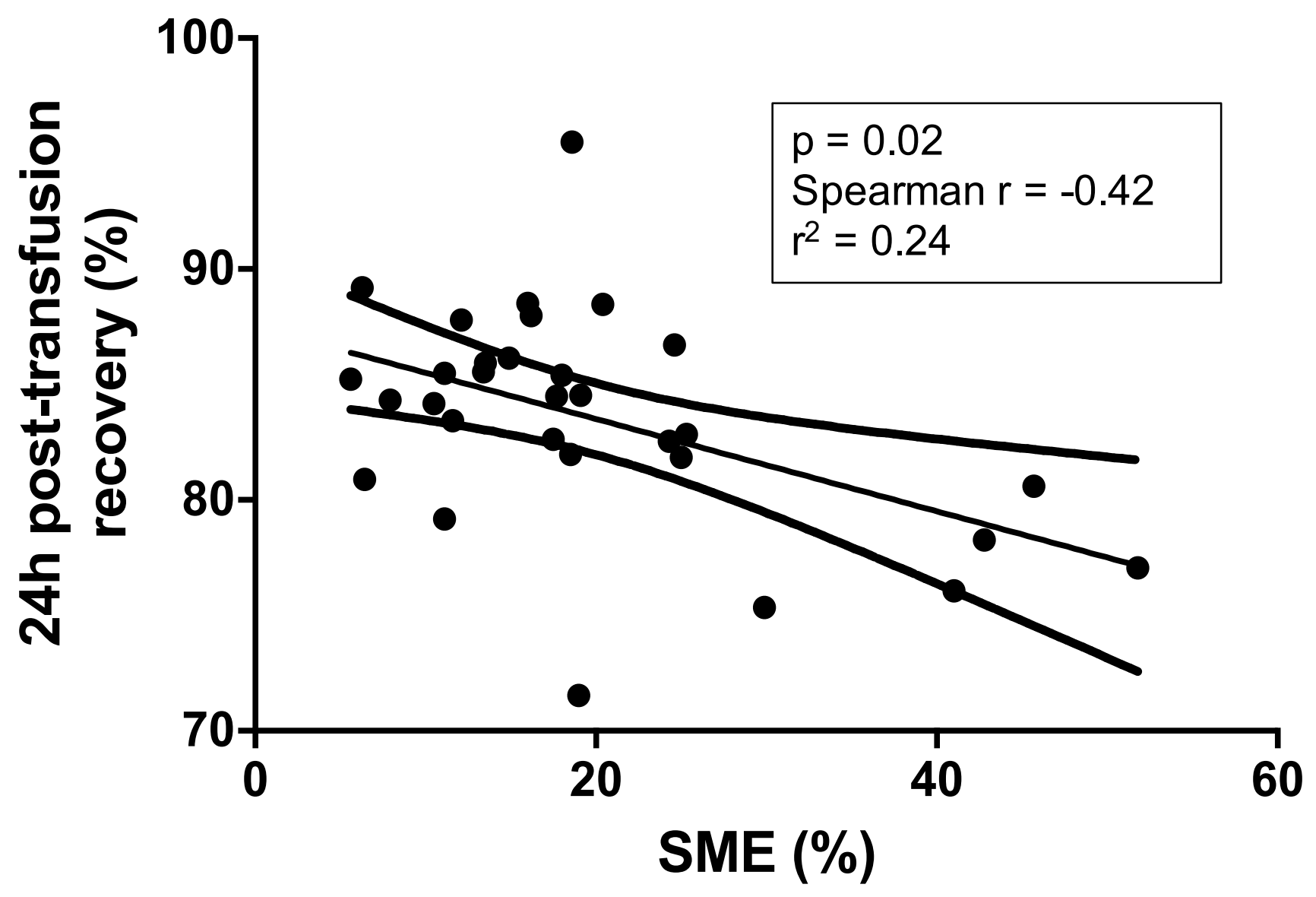

Figure 2 
A

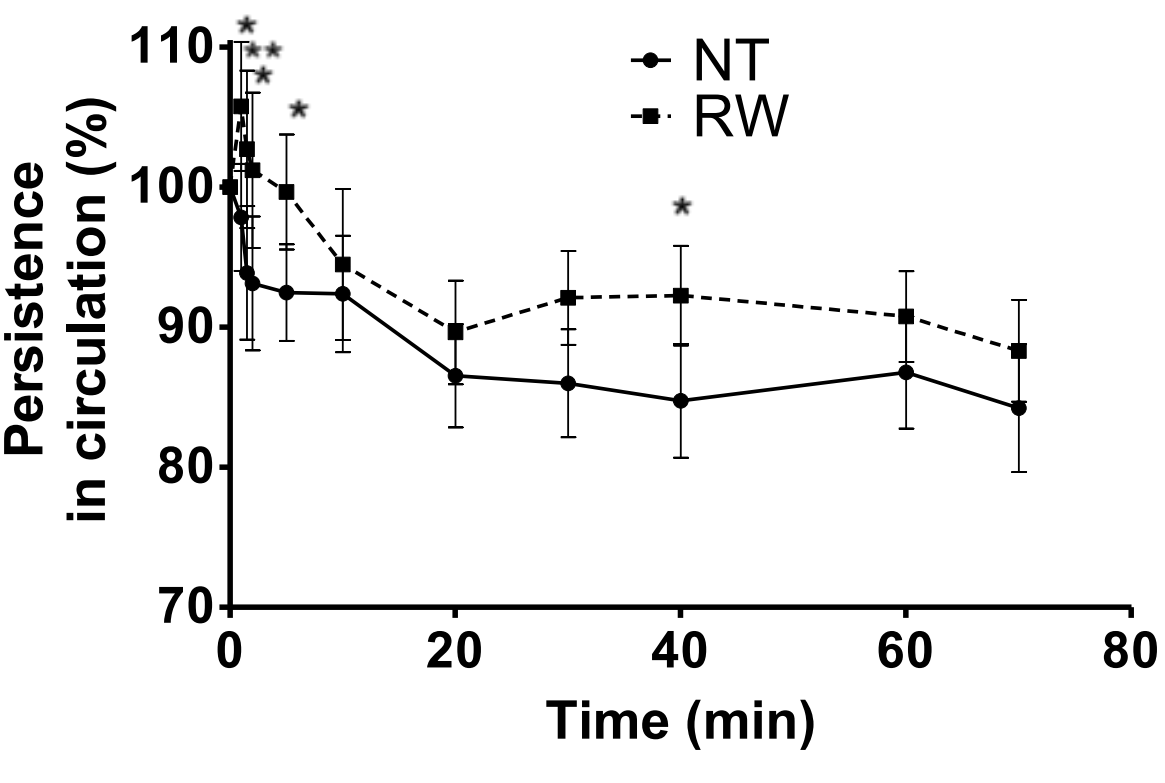

C

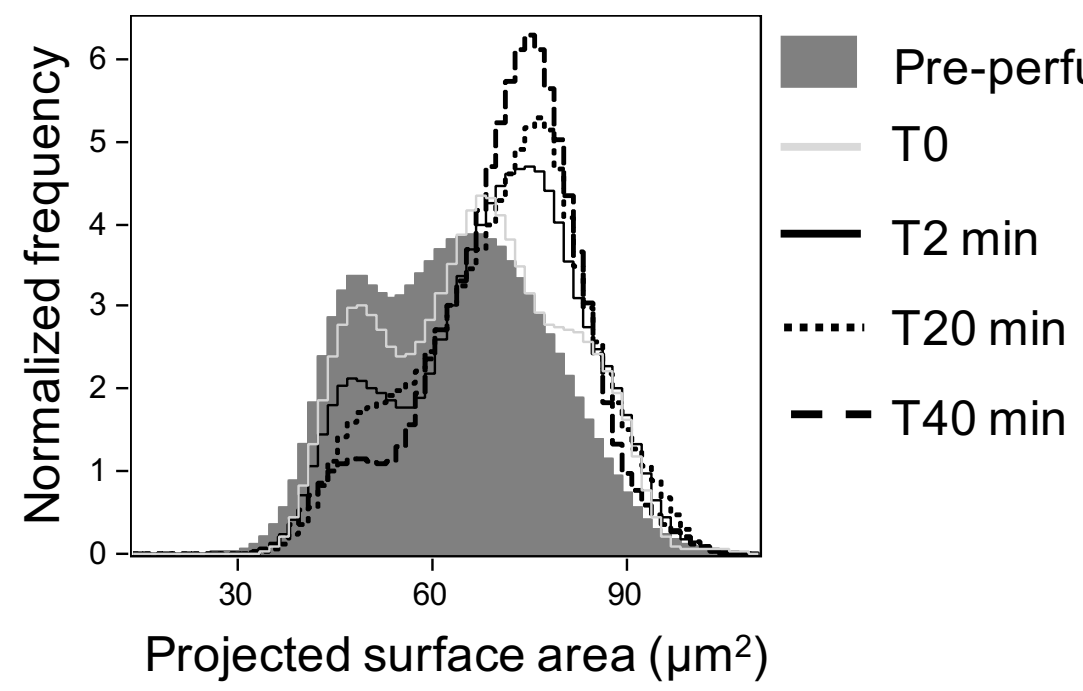

$E$

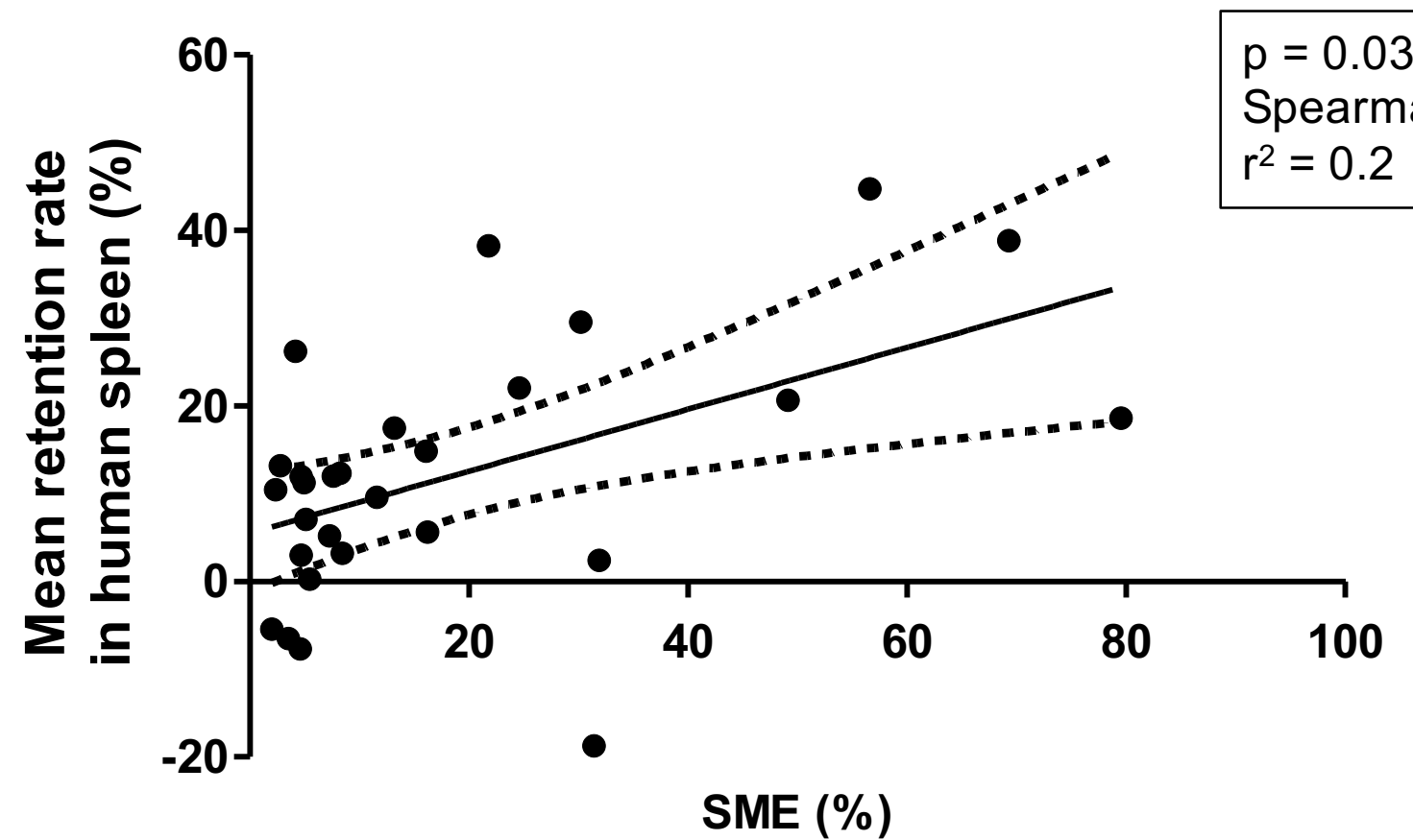

B

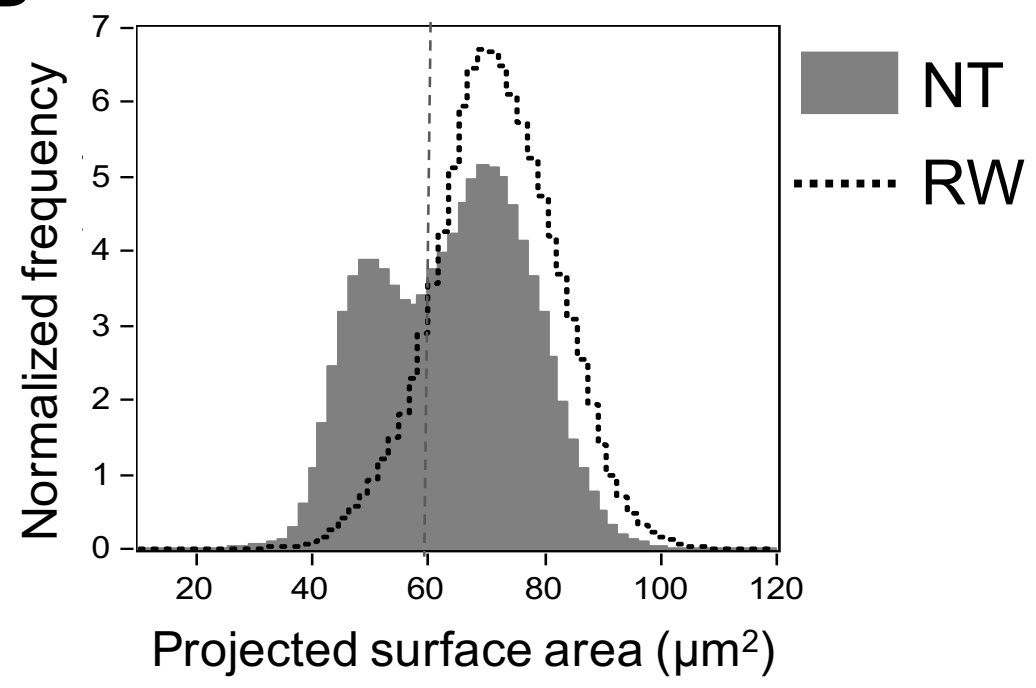

D

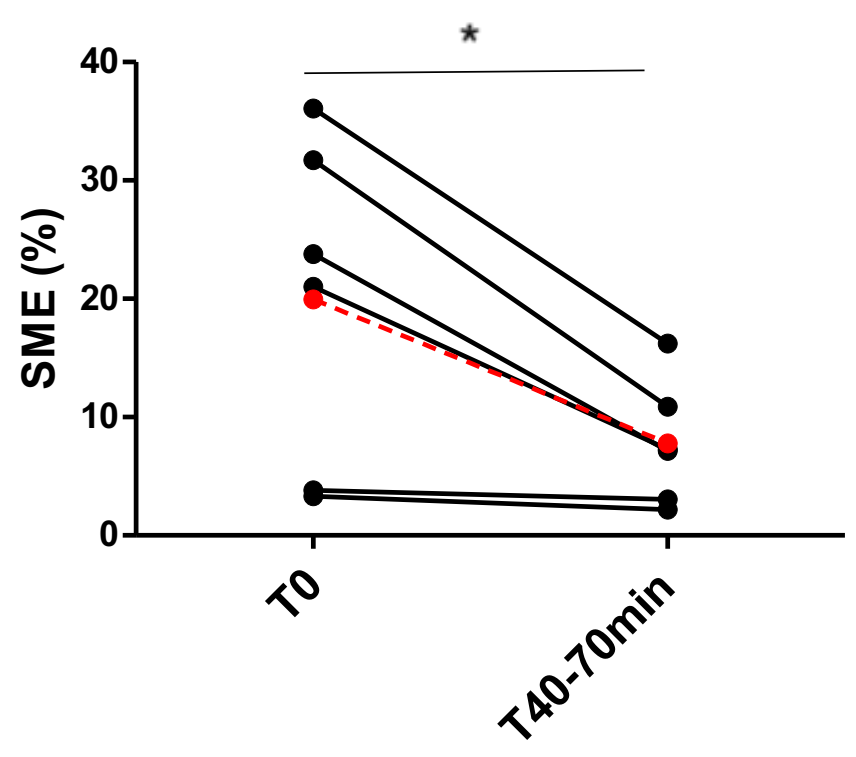

$p=0.03$

Spearman $r=0.5$

$\mathrm{r}^{2}=0.2$ 
A

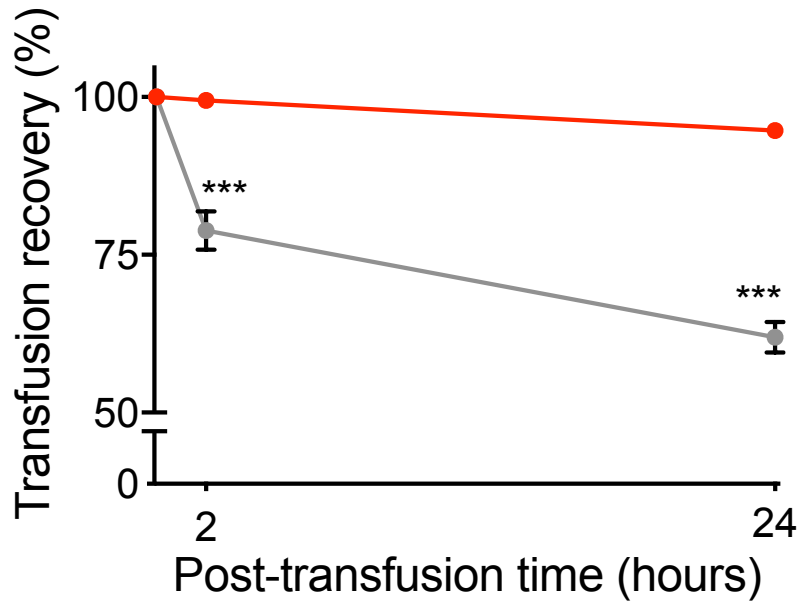

B

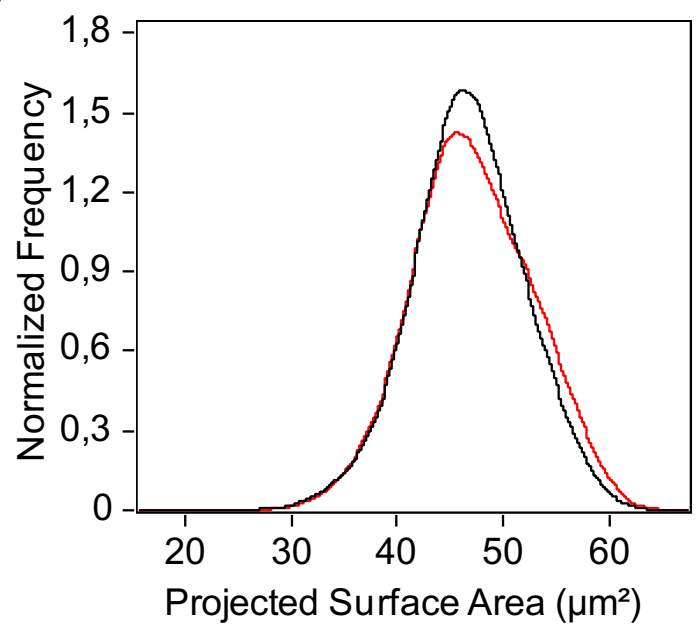

C

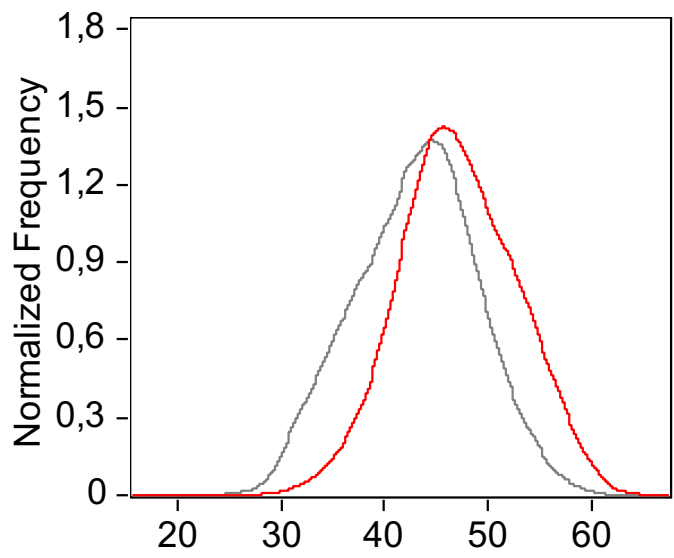

Projected Surface Area $\left(\mu \mathrm{m}^{2}\right)$
D

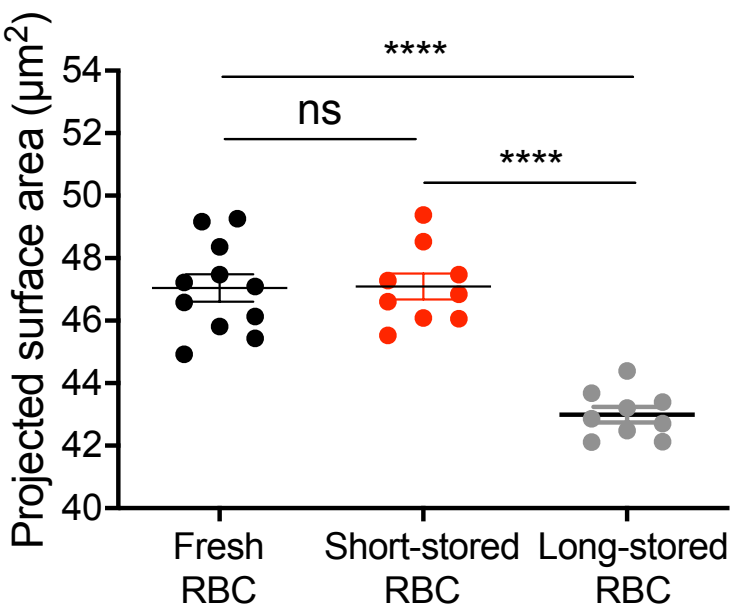

$E$

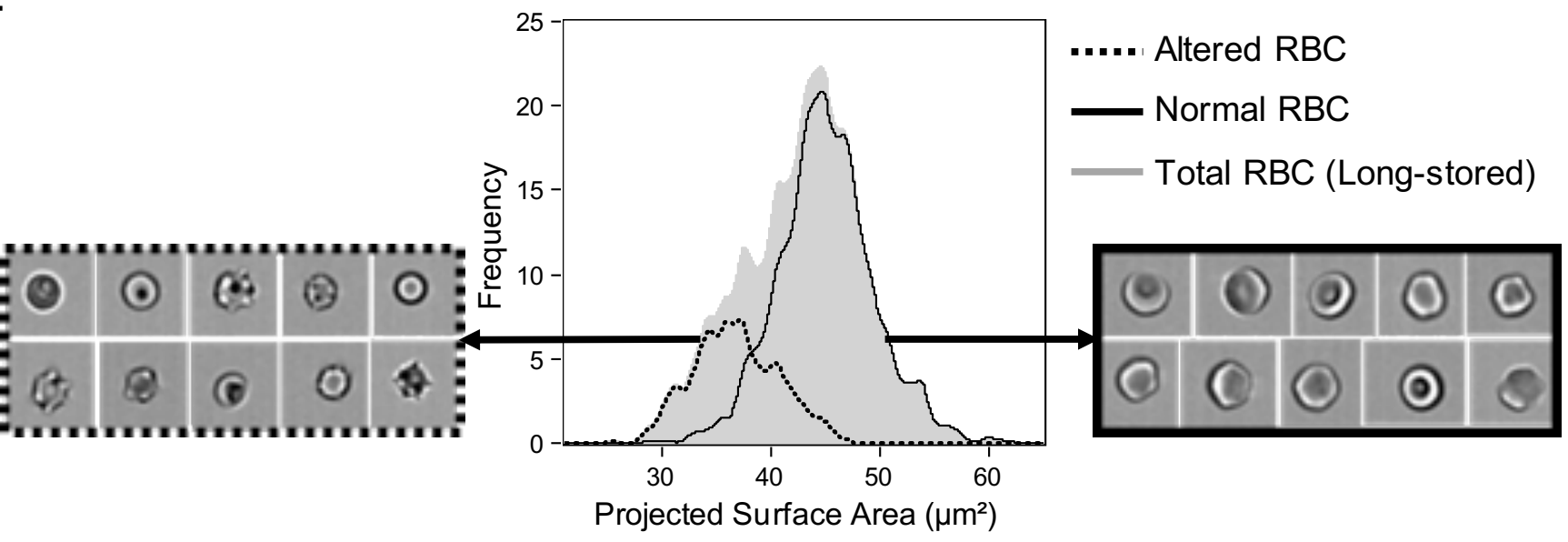

F

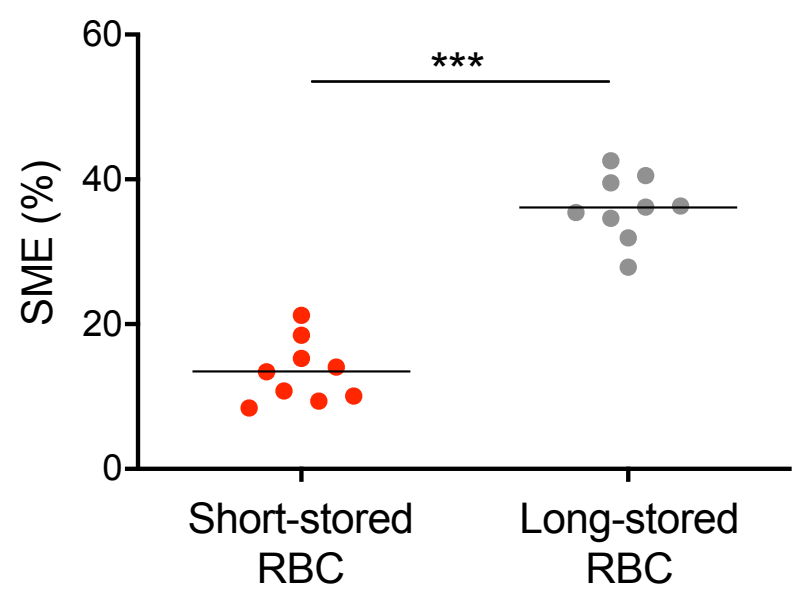




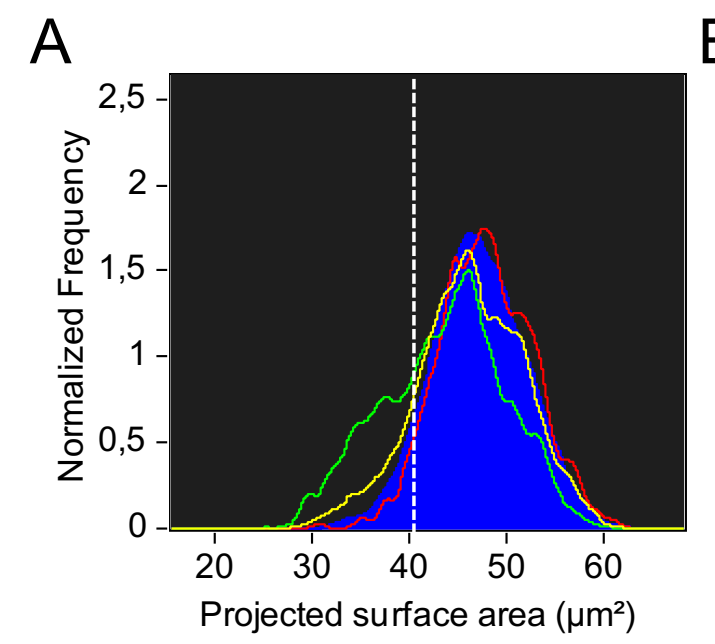

D

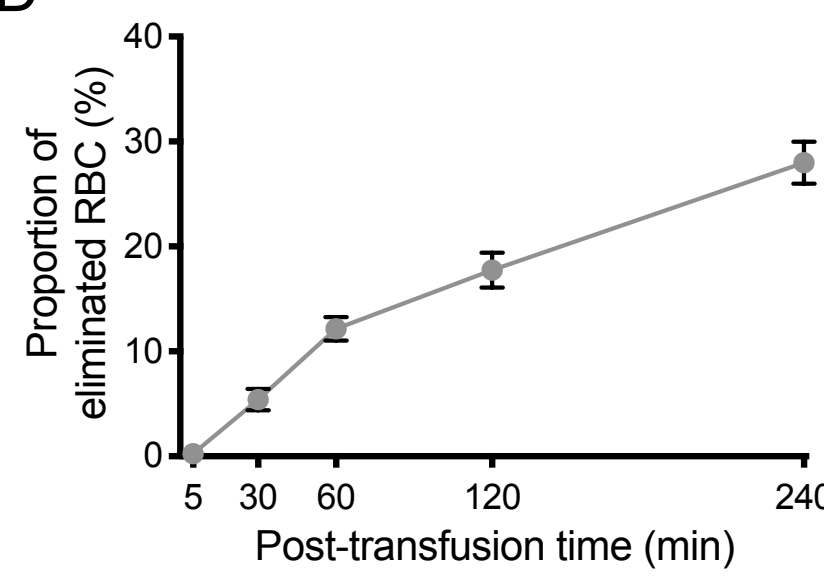

F1

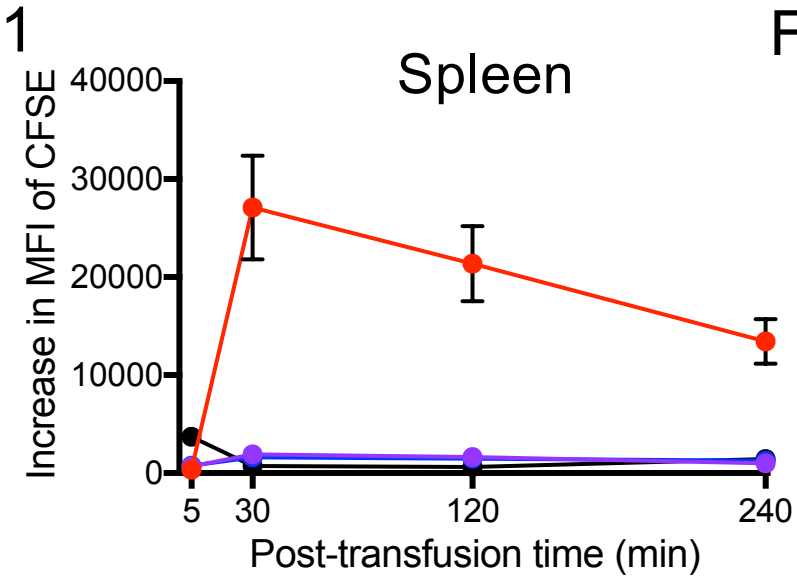

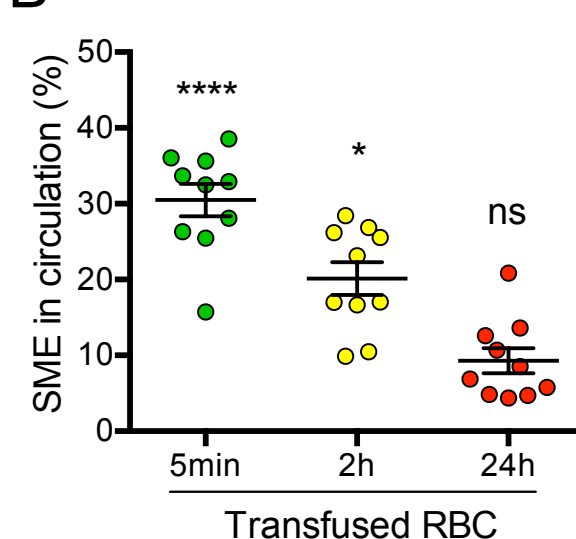

from recipient's circulation
C

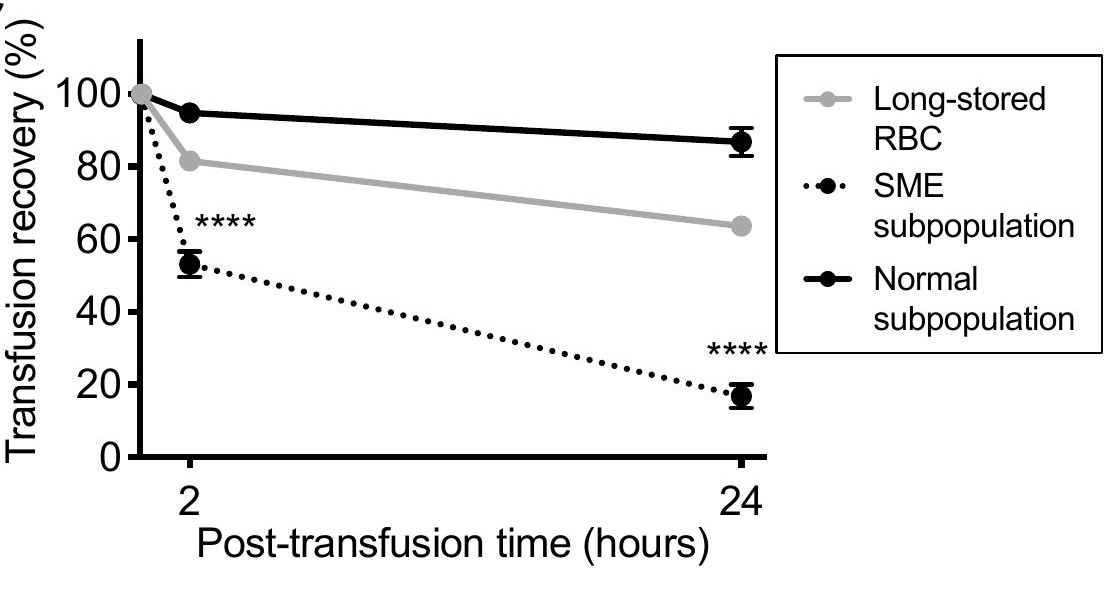

E

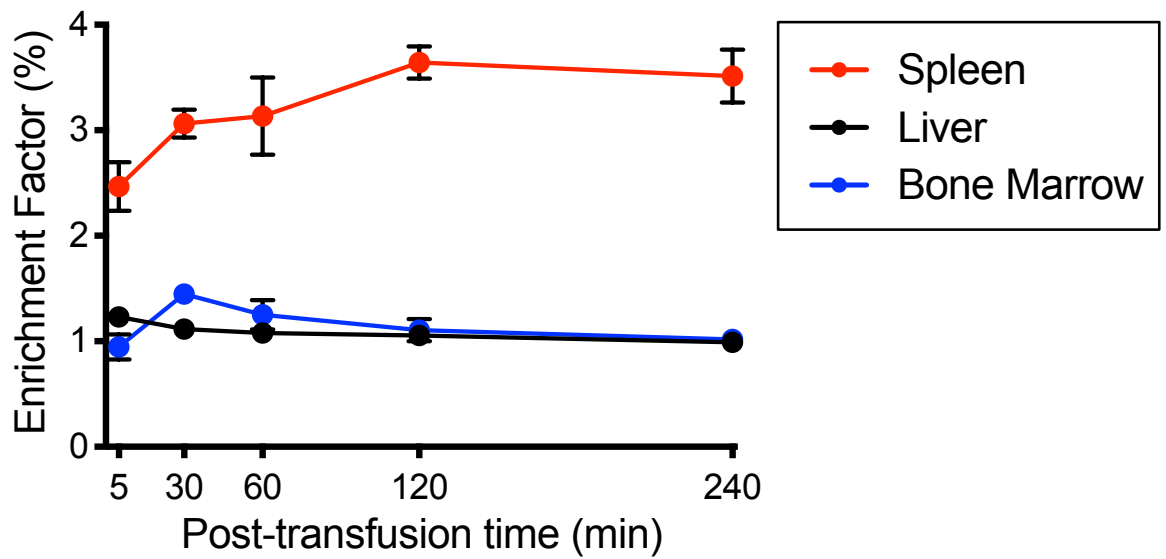

F2

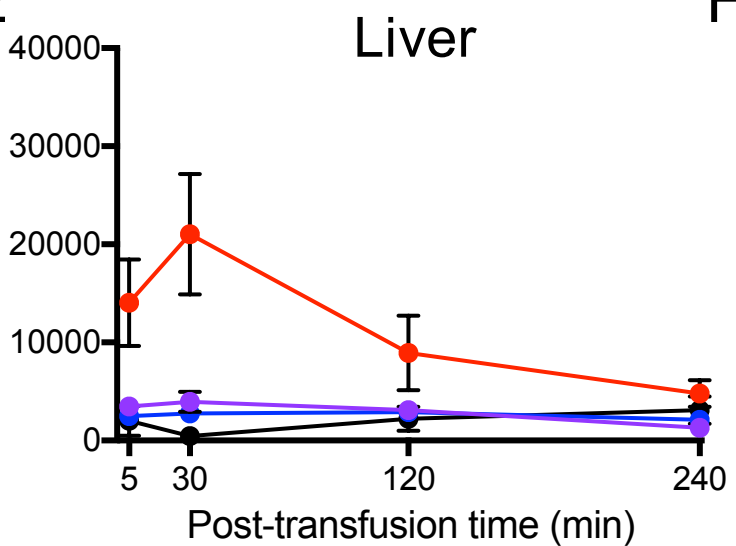

F3

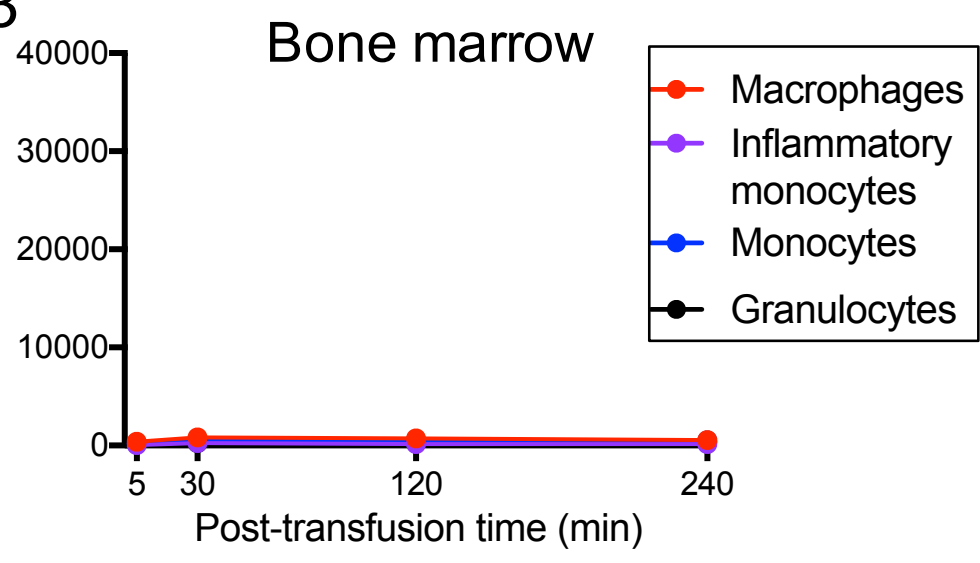


A

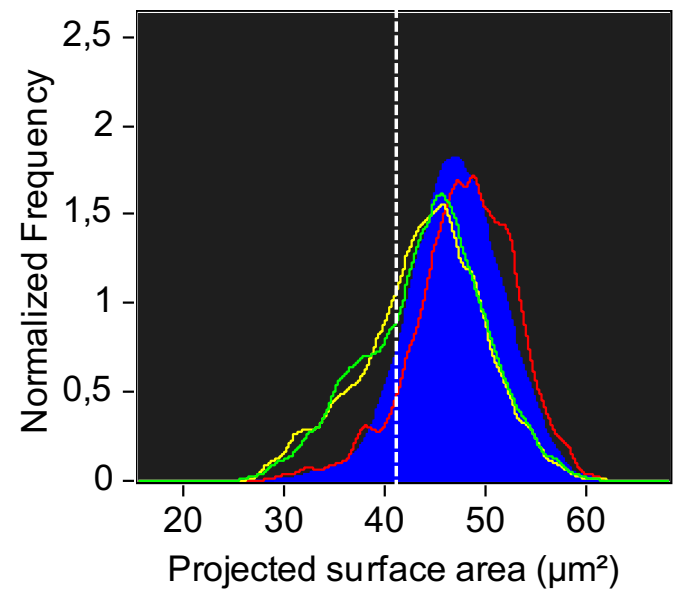

C

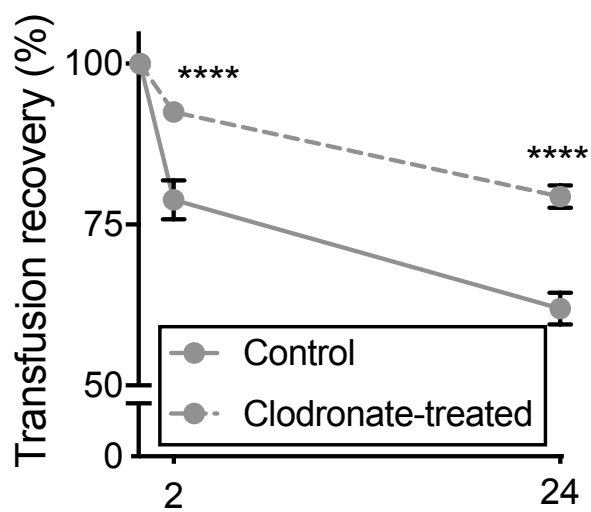

Post-transfusion time (hours)

E

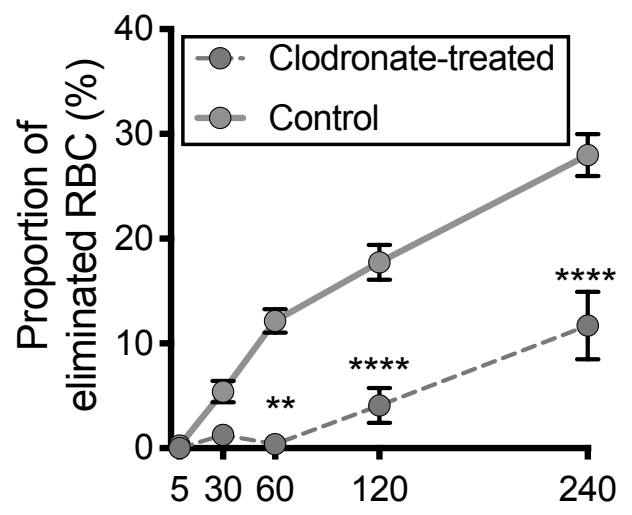

Post-transfusion time (min)

G1

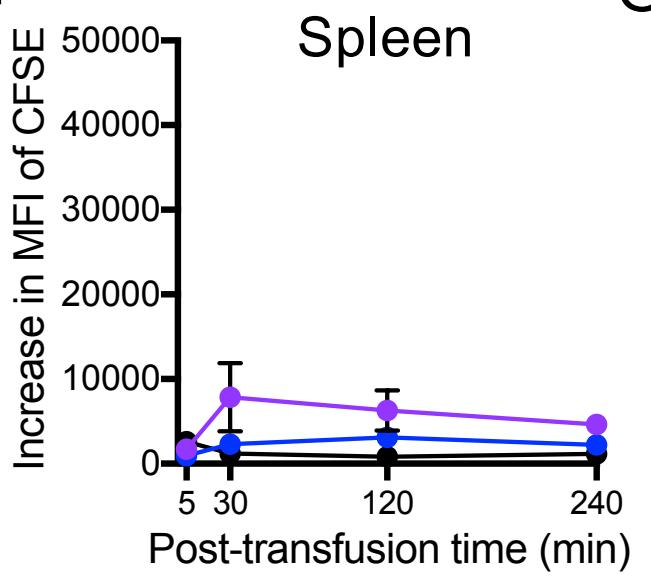

B
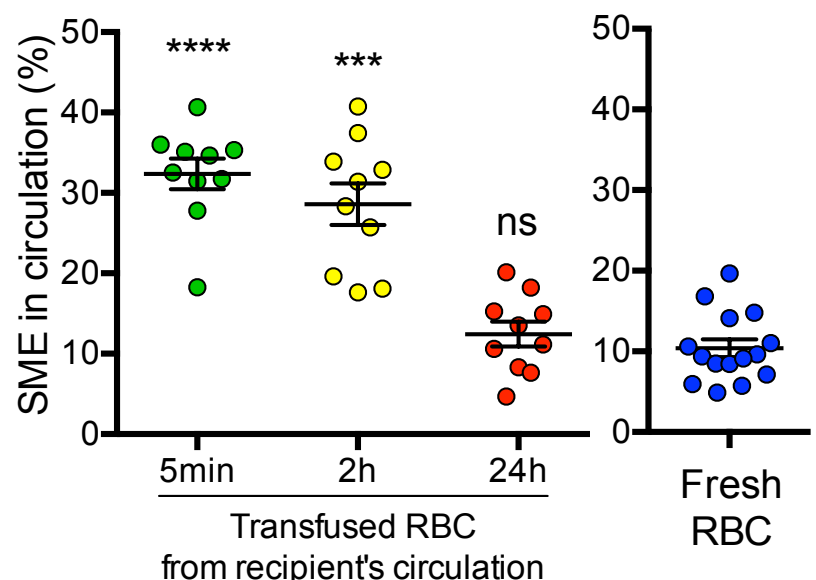

D

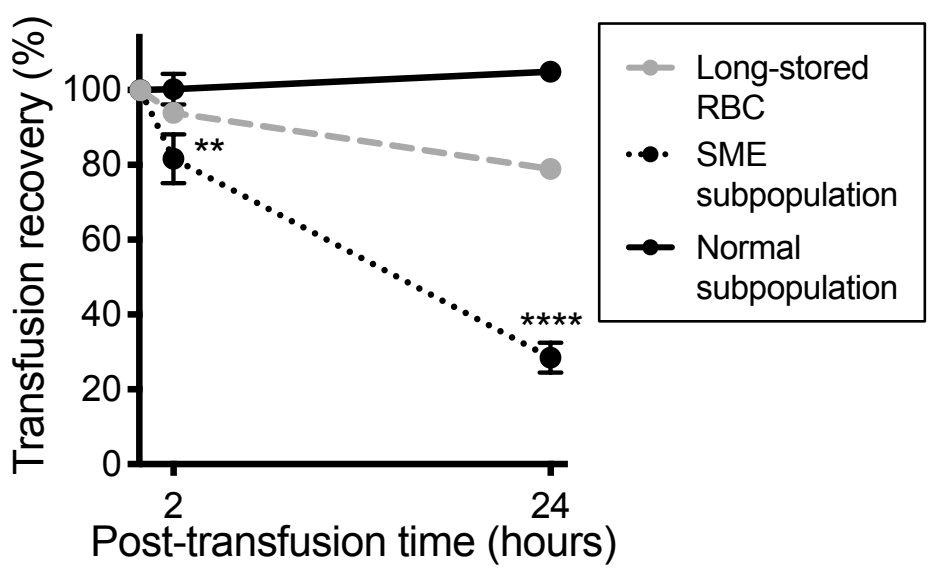

F

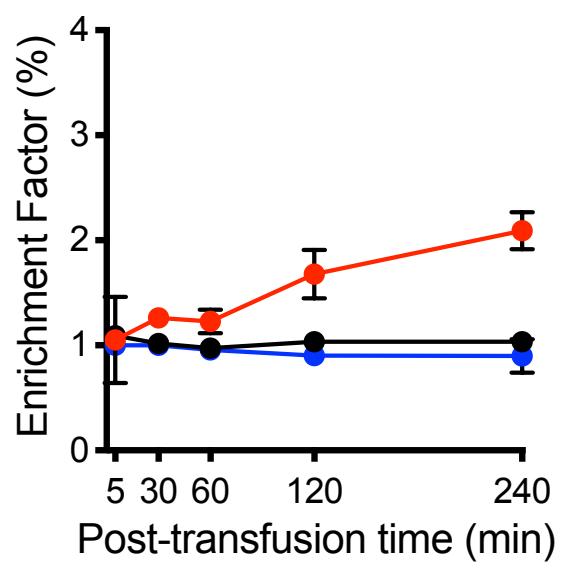

G2

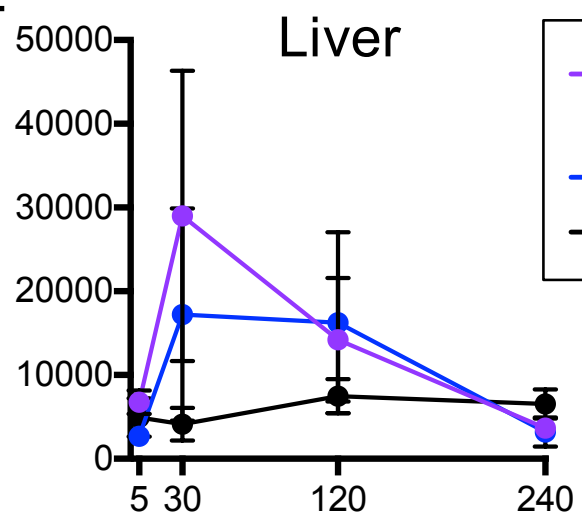

Post-transfusion time (min) 
A

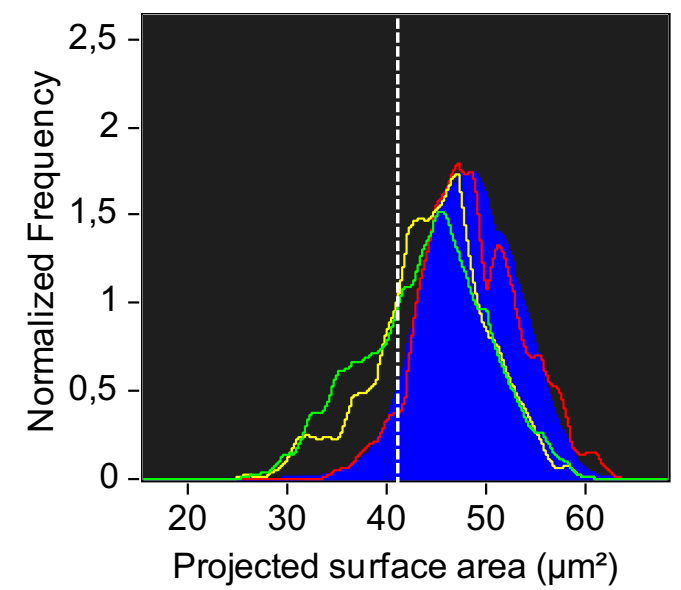

C

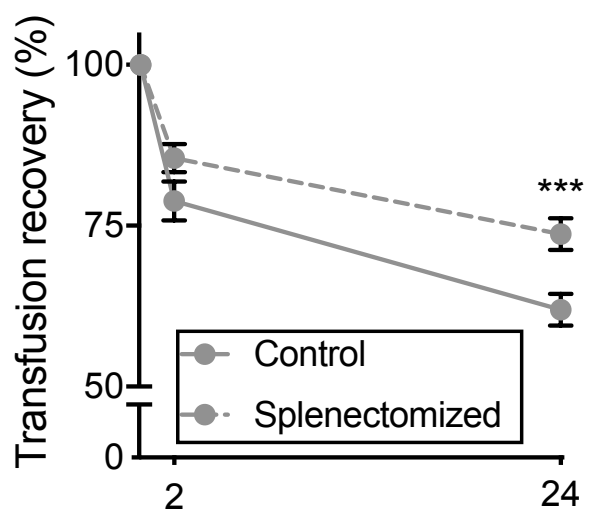

Post-transfusion time (hours)

$E$

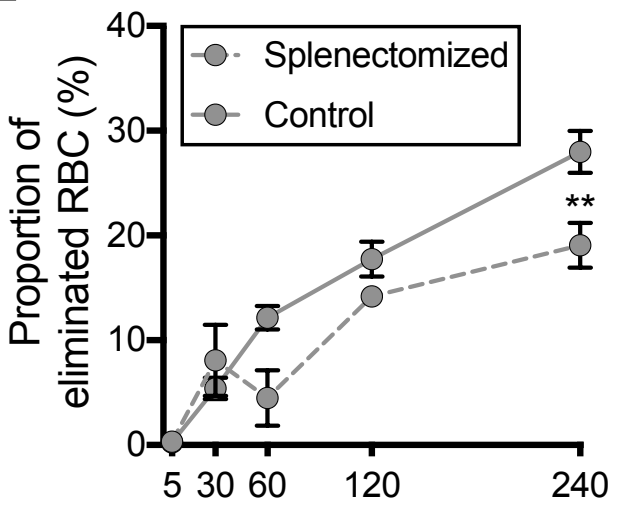

Post-transfusion time (min)

\section{G1}

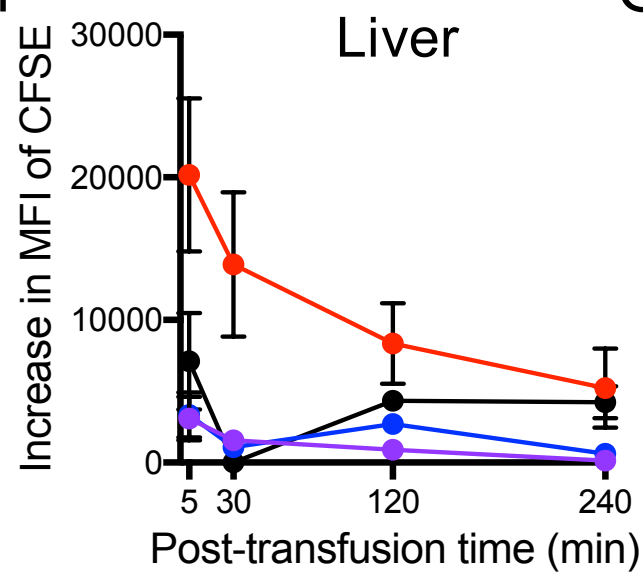

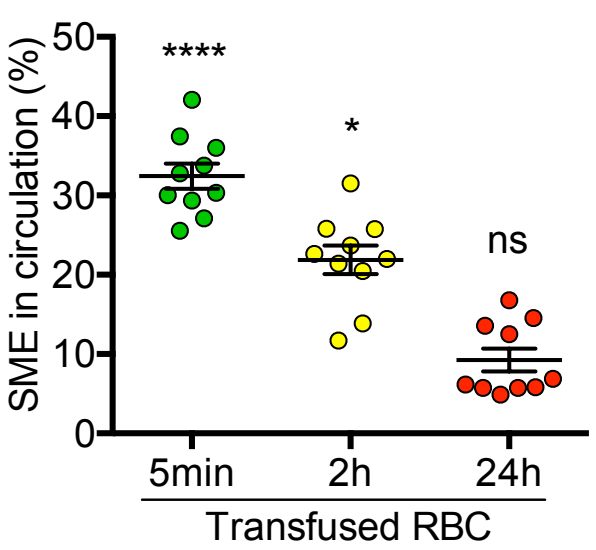

from recipient's circulation

D

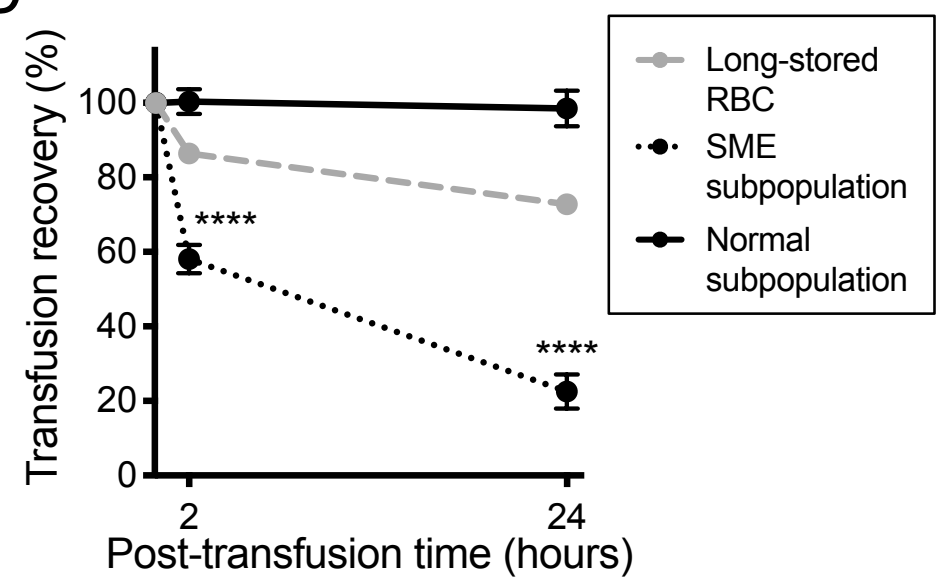

$\mathrm{F}$

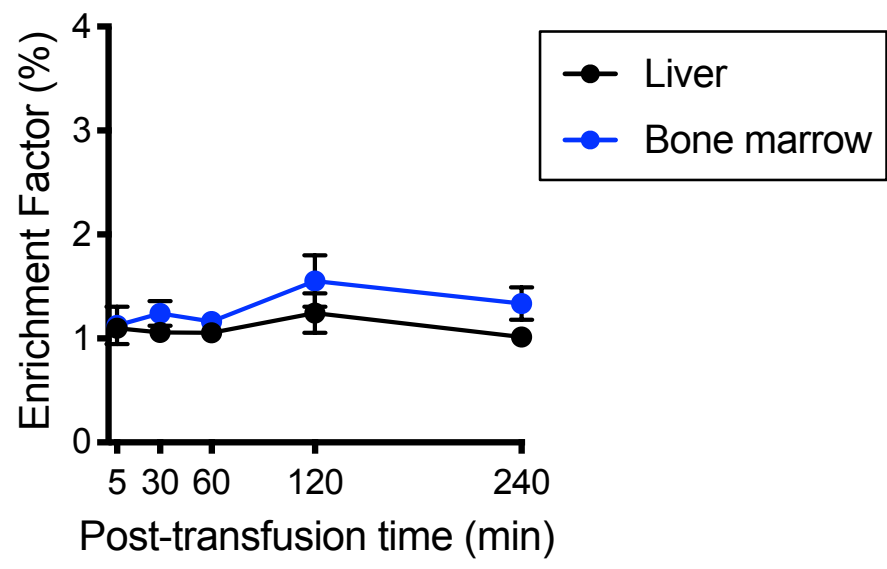

G2

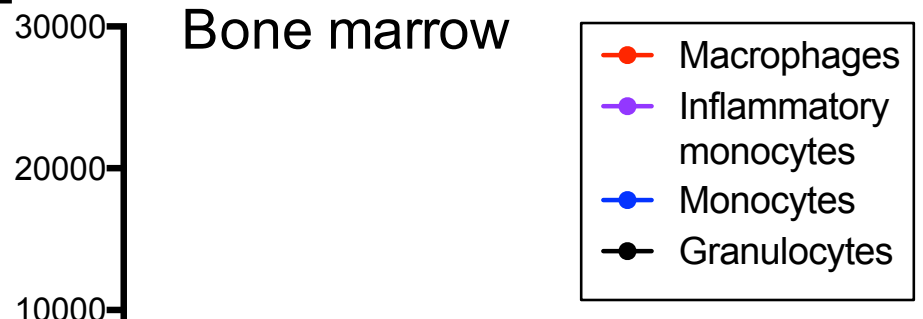

10000

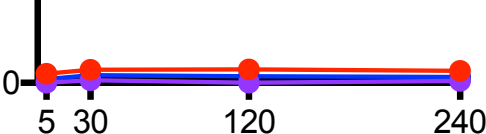

Post-transfusion time (min) 


\section{Supplementary data}

\section{Supplementary methods \\ $R B C$ concentrates collection and storage}

SAGM units were collected using a five-fold NPT blood bag system (Macopharma, Tourcoing, France) with LEUCOFLEX LCRD2 leukoreduction filter was used for collection, leukofiltration of whole blood and separation of plasma. RBC concentrates were obtained from routine blood center inventory $(n=17)$ or were deemed not suitable for clinical use $(\mathrm{n}=18)$. In the latter case, blood was specifically donated for non-therapeutic use by donors with contraindications for allogeneic blood donation (e.g., recent tattoo or piercing, travel, dental care).

AS-3 units were collected using the Leukotrap ${ }^{\circledR}$ RC System with RC2D Filter (Haemonetics). The AS-1 unit was collected using BIOFLEX RC Red Cell Leukocyte reduction filter in PL2209 plastic bags (Fenwal). Both used the red cell filtration method.

\section{Imaging flow cytometry analysis on human samples}

Imaging flow cytometry using ImageStream X Mark II (AMNIS part of EMD Millipore) was performed to determine RBC dimensions and morphology by using brightfield images (60X magnification) processed with computer software (IDEAS v6.2, AMNIS). Focused cells and single cells were respectively selected using the features gradient RMS and aspect ratio versus area. Front views were selected and analyzed by the mask "Object" and the features of circularity, perimeter and area. At least 6000 front views of focused single RBC/condition were analyzed. SME proportion was determined independently for each donor, using the nadir of the bimodal frequency histograms as the gating boundary. Stored RBC were suspended at $1 \%$ hematocrit just before acquisition (INSPIRE software, AMNIS) in a Krebs-albumin solution (Krebs-Henseleit buffer, Sigma-Aldrich) modified with $2 \mathrm{~g}$ of glucose, $2.1 \mathrm{~g}$ of sodium bicarbonate, $0.175 \mathrm{~g}$ of calcium chloride dehydrate, and $5 \mathrm{~g}$ of lipid-rich bovine serum albumin (Albu-MAX II, Thermo Fisher Scientific) for $1 \mathrm{~L}$ of sterile water ( $\mathrm{pH} 7.4$ ).

\section{In vivo erythrophagocytosis}

Spleen and liver cells were dissociated mechanically using dedicated dissociation kits and gentleMACS dissociator (Miltenyi Biotec). Bone marrow cells were obtained by flushing tibia and femur with $\mathrm{PBS} / 2 \% \mathrm{FBS} / \mathrm{EDTA} 2 \mathrm{mM}$. RBC were removed from the suspension using ACK lysing buffer (Invitrogen). Cells were first incubated with anti-mouse CD16/CD32 clone $2.4 \mathrm{G} 2$ to block IgG receptors (BD). Then, cells were stained at $4{ }^{\circ} \mathrm{C}$ using a panel of antibodies.

\begin{tabular}{|l|l|l|l|}
\hline Antibody & Clone & Fluorochrome & Provider \\
\hline CD3 $\varepsilon$ & $145-2 C 11$ & APC & Sony \\
\hline CD19 & $6 D 5$ & APC & Sony \\
\hline NK1.1 & PK136 & APC & Sony \\
\hline F4/80 & BM8 & eF450 & Ebioscience \\
\hline CD11b & M1/70 & AF700 & BD \\
\hline Ly-6G & 1A8 & PerCP & Sony \\
\hline Ly-6C & HK1.4 & PeCy7 & Sony \\
\hline
\end{tabular}


Macrophages (CD3 $\left./ \mathrm{CD} 19^{-} / \mathrm{NK} 1.1^{-} / \mathrm{Ly}-6 \mathrm{G}^{-} / \mathrm{F} 4 / 80^{\mathrm{hi}} / \mathrm{CD} 11 \mathrm{~b}^{\mathrm{lo}}\right)$, monocytes $\left(\mathrm{CD} 3^{-} / \mathrm{CD} 19^{-}\right.$

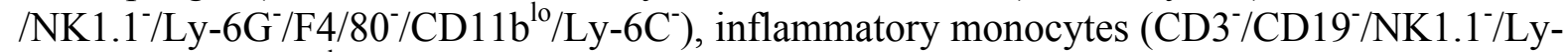
$\left.6 \mathrm{G}^{-} / \mathrm{F} 4 / 80^{-} / \mathrm{CD} 11 \mathrm{~b}^{\mathrm{lo}} / \mathrm{Ly}-6 \mathrm{C}^{+}\right)$, and granulocytes $\left(\mathrm{CD}^{-} / \mathrm{CD} 19^{-} / \mathrm{NK} 1.1^{-} / \mathrm{Ly}-6 \mathrm{G}^{+}\right)$were analyzed. Stained samples were analyzed by flow cytometry and background fluorescence from nontransfused mice was subtracted for each cell type. 


\section{Supplementary figures}
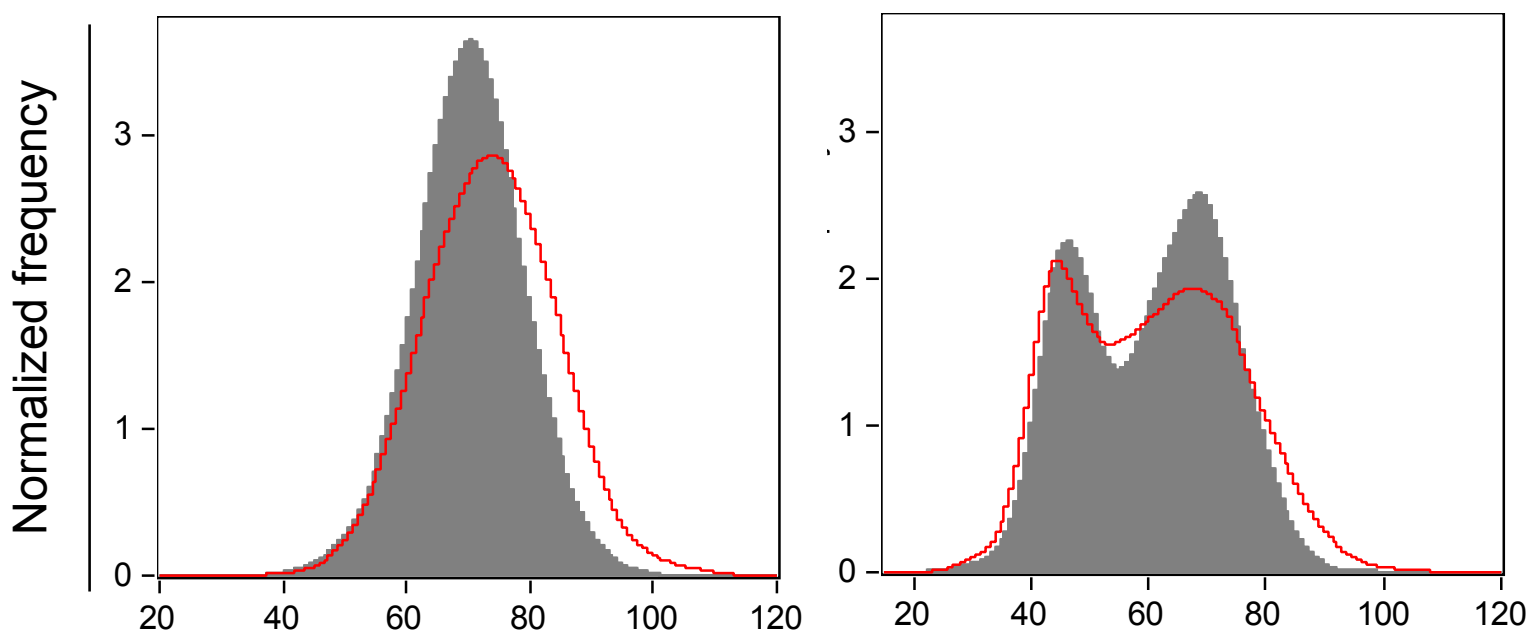

Projected surface area $\left(\mu \mathrm{m}^{2}\right)$

Supplementary Figure 1: RBC morphology after resuspension in fresh plasma. Projected surface area on normalized frequency plot for fresh (left panel) or stored RBC (right panel) is similar when a Krebs-albumin solution (in gray) or neat fresh plasma (in red) is used as a resuspension medium for imaging flow cytometry analysis (15min incubation before acquisition) . 

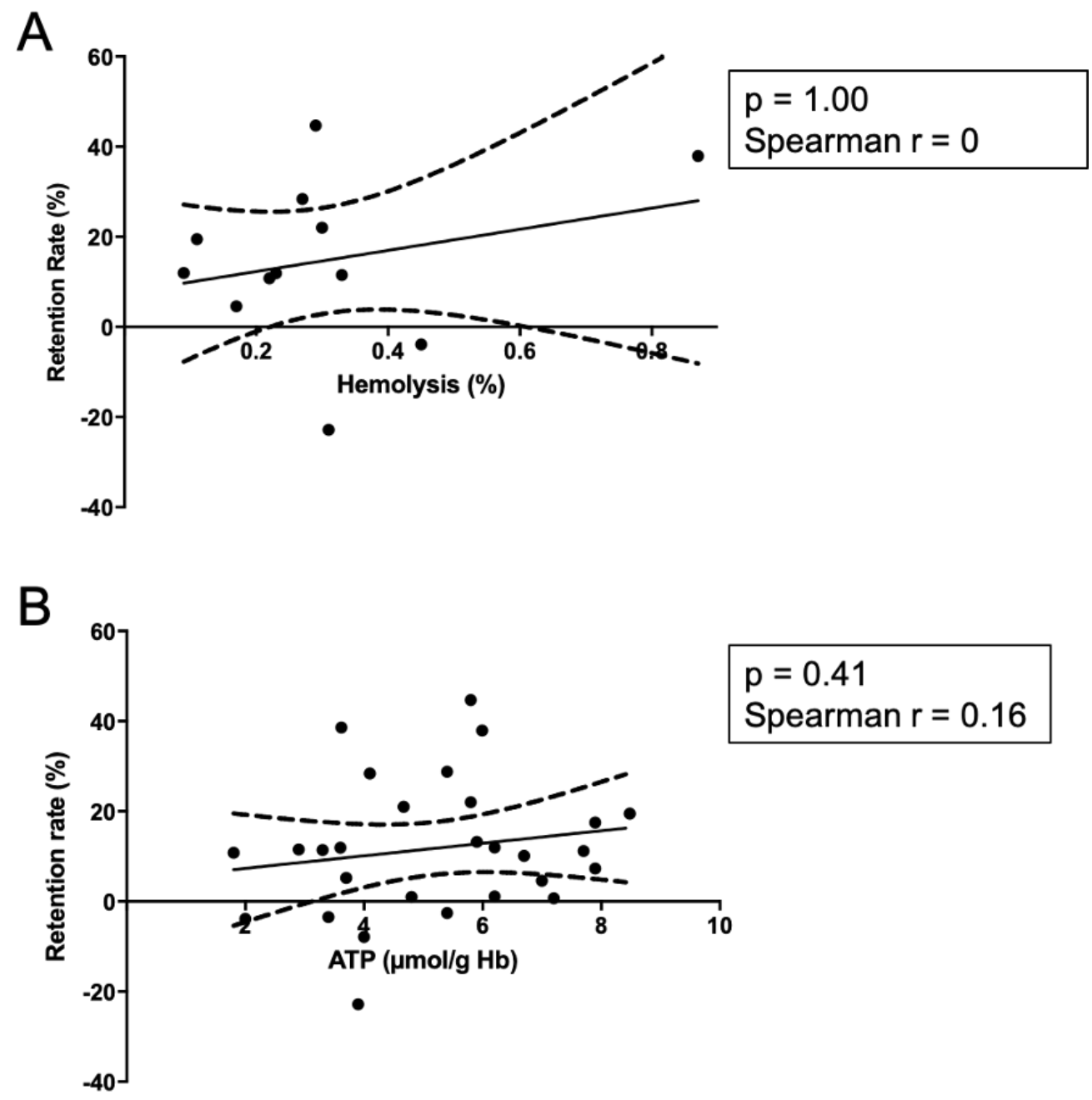

$$
\begin{aligned}
& p=0.41 \\
& \text { Spearman } r=0.16
\end{aligned}
$$

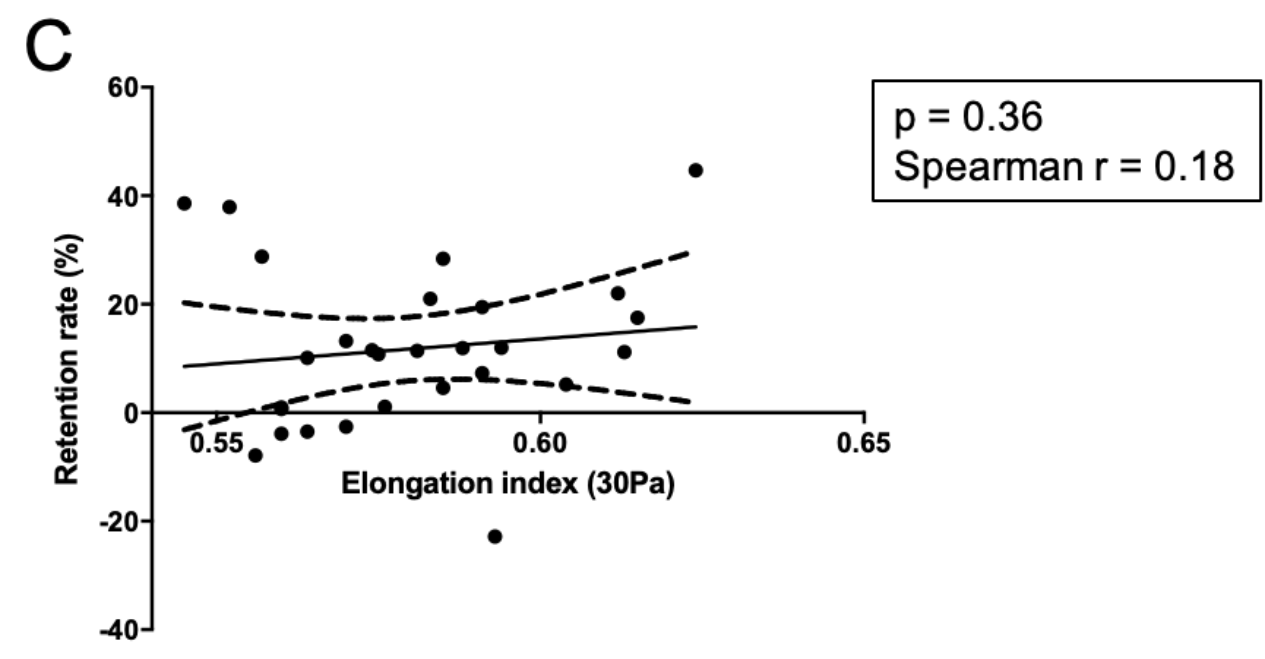

Supplementary Figure 2: Correlation between spleen retention rate and classical markers of the storage lesion. Correlation between mean retention rate in human spleens perfused ex vivo and storage hemolysis (A), intracellular ATP level (B), and elongation index measured at 30 Pascal $(\mathrm{Pa})$ by LORRCA $(\mathrm{C})$ in the $\mathrm{RBC}$ concentrate before perfusion is shown. 


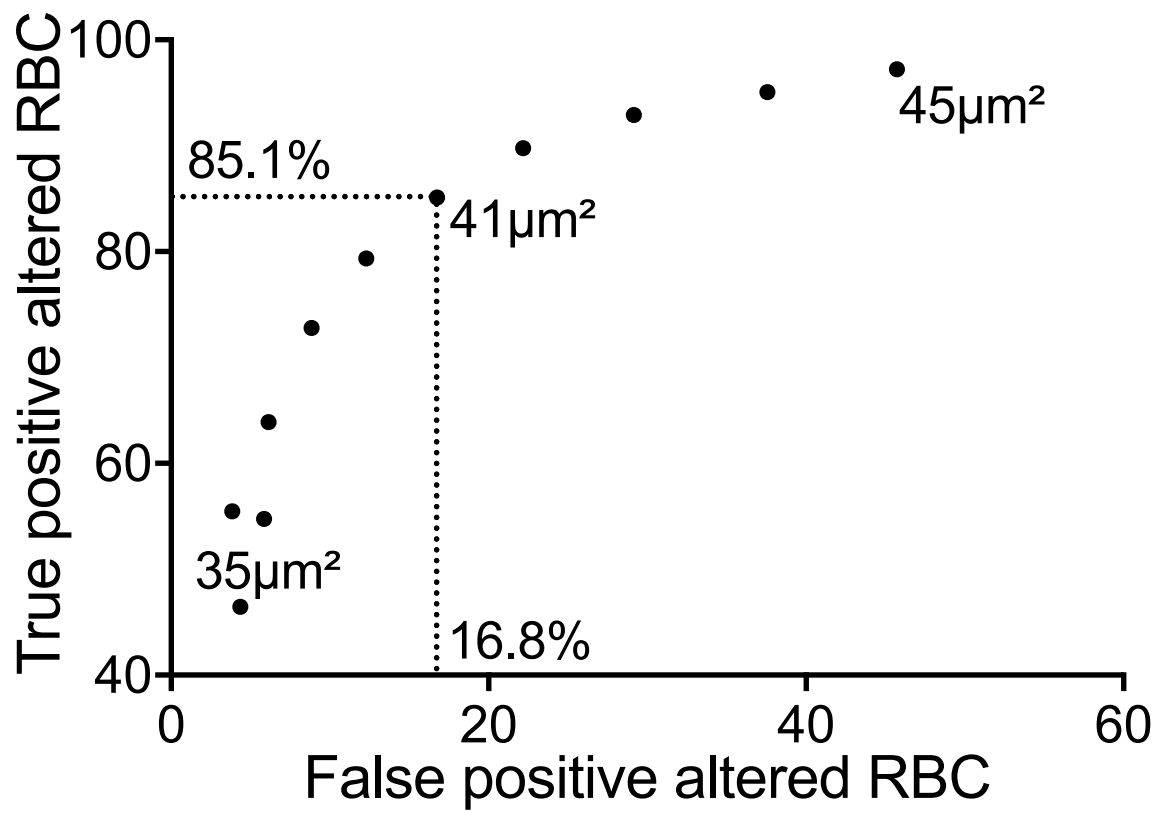

Supplementary Figure 3: ROC analysis to determine optimal cutoffs for quantifying the proportion of morphologically altered mouse RBC by Imaging Flow Cytometry. A

threshold set at $41 \mu \mathrm{m}^{2}$ enables identification of altered RBC with a specificity of $83.2 \%$ and a sensitivity of $85.1 \%$. 

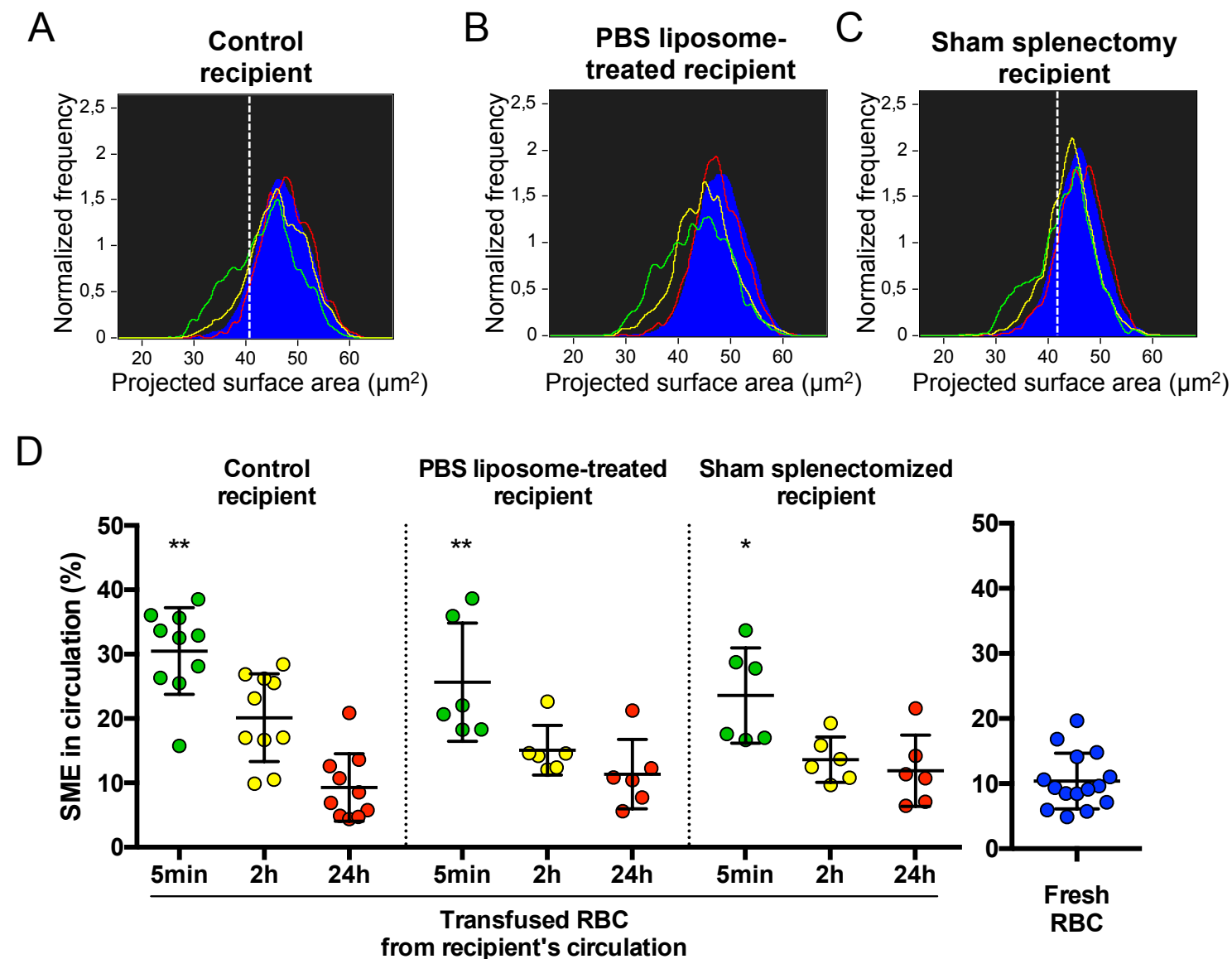

E
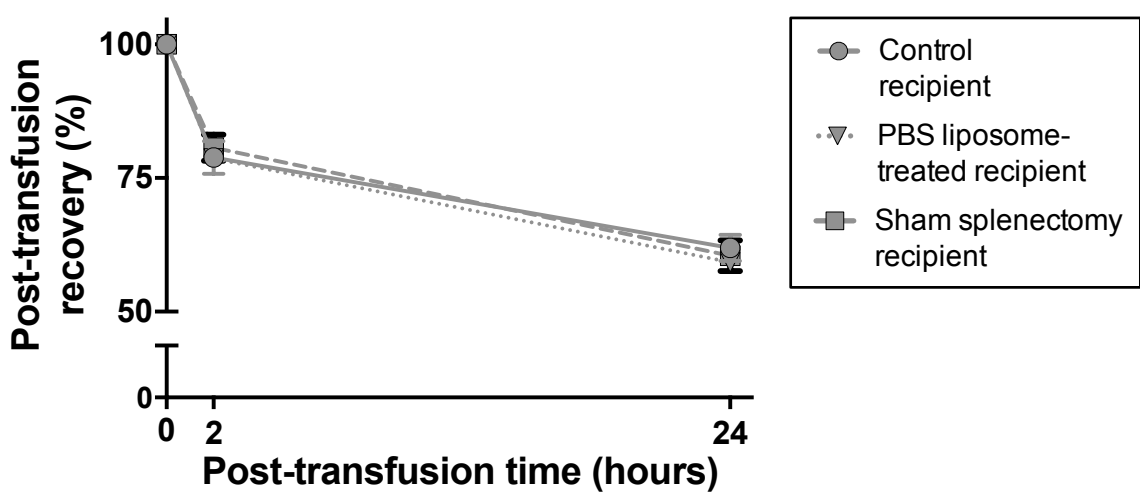

Supplementary Figure 4: Comparison of the transfused RBC morphology and transfusion recovery in the circulation of different control recipient mouse subgroups.

Representative normalized frequency plot of projected surface area for long-stored mouse $\mathrm{RBC}$, as observed at $5 \mathrm{~min}$ (green line), $2 \mathrm{~h}$ (yellow line), and $24 \mathrm{~h}$ (red line) after transfusion to a control (A), PBS liposome-treated recipients (B) or sham splenectomized (C). Control $\mathrm{RBC}$ from a non-transfused mouse (blue) are shown as a reference. The dashed white vertical line defines the gating of SME. (D) Declining proportion of SME in the circulation following transfusion is similar in sham splenectomized, PBS liposome-treated and control recipients (n = a minimum of 6 mice/group). (E) Transfusion recovery is similar in sham splenectomized, PBS liposome-treated and control recipients ( $\mathrm{n}=\mathrm{a}$ minimum of 6 mice/group). In $\mathrm{D}$ and $\mathrm{E}$, data are presented as mean \pm SEM. In $\mathrm{D},{ }^{*} \mathrm{P}<0,05,{ }^{*} \mathrm{P}<0,01$ when compared to the "Fresh RBC" condition by a Kruskal-Wallis test. 